\title{
11. PALEOENVIRONMENTAL SIGNIFICANCE OF CLAY MINERALOGICAL AND GEOCHEMICAL DATA, SOUTHWEST ATLANTIC, DEEP SEA DRILLING PROJECT LEGS 36 AND 71
}

\author{
Christian Robert, Géologie Marine, L.A. CNRS 41, Faculté des Sciences de Luminy, Case 901, \\ 13288 Marseille Cedex 9, France \\ and \\ Henri Maillot, Sédimentologie et Géochimie, E.R.A. CNRS 764, Université de Lille 1, \\ 59655 Villeneuve d'Ascq Cedex, France
}

\begin{abstract}
For Middle Jurassic to Pleistocene times, clay mineralogical and geochemical data provide information on the evolution of continental and marine paleoenvironments. They are a source of information on marginal instability, on the continental and shallow marine environments related to the development of the Southern Ocean during the Middle and Late Jurassic, and on tectonic relaxation of the continental margins at the end of the Late Jurassic. They also provide evidence for the influences of the South Atlantic opening and the movement of the Falkland Plateau in a reduced marine environment until Aptian-Albian times, and the transition to an open marine environment during Albian time; the influences of the Albian-Turonian and Coniacian-Santonian Andean deformations in an open marine environment; the limited tectonic effects and strong influence of marine currents at the Cretaceous/Tertiary boundary; the influences of the global climatic cooling and inferred bottom water circulation during the late Eocene and Oligocene; the widening of the South Atlantic Ocean during Oligocene time, which was accompanied by an increased influence of the biogenic components on sedimentation; increased carbonate dissolution from late Oligocene to early Miocene, related to the deepening of the ocean; limited mineralogical and important geochemical modifications when the Drake Passage opened in the early Miocene; the influence of the late Miocene development of the Antarctic ice-sheet; the major Antarctic cooling and Patagonian glaciation during Pliocene time; and the change in the Antarctic Bottom Water circulation at the Pliocene/Pleistocene boundary.
\end{abstract}

\section{INTRODUCTION}

During Legs 36 and 71 of the Deep Sea Drilling Project, holes were drilled at eight sites on the Falkland Plateau, in the Georgia Basin, and on the western flank of the Mid-Atlantic Ridge (Fig. 1). The sediments recovered range from Pleistocene to Jurassic. Site characteristics are summarized in the list.

\begin{tabular}{|c|c|c|c|c|c|c|}
\hline \multirow[b]{2}{*}{ Hole } & \multirow{2}{*}{$\begin{array}{l}\text { Position } \\
\text { (Latitude; Longitude) }\end{array}$} & \multirow{2}{*}{$\begin{array}{c}\text { Water } \\
\text { Depth } \\
\text { (m) }\end{array}$} & \multirow{2}{*}{$\begin{array}{l}\text { Penetr. } \\
\text { (m) }\end{array}$} & \multicolumn{2}{|c|}{ No. Samples } & \multirow{2}{*}{$\begin{array}{l}\text { Oldest } \\
\text { Sediment } \\
\text { Recovered }\end{array}$} \\
\hline & & & & Min. & Geochem. & \\
\hline \multicolumn{7}{|l|}{ Leg 36} \\
\hline $327,327 \mathrm{~A}$ & $50^{\circ} 52.28^{\prime} \mathrm{S} ; 46^{\circ} 47.02^{\prime} \mathrm{W}$ & 2400 & 470 & 66 & 63 & \multirow{4}{*}{$\begin{array}{l}\text { Neocomian } \\
\text { Turonian } \\
\text { Paleocene } \\
\text { Jurassic } \\
\text { on basement }\end{array}$} \\
\hline $328,328 \mathrm{~A}, \mathrm{~B}$ & $49^{\circ} 48.67^{\prime} \mathrm{S} ; 36^{\circ} 39.53^{\prime} \mathrm{W}$ & 5095 & 471 & 54 & & \\
\hline 329 & $50^{\circ} 39.31^{\prime} \mathrm{S} ; 46^{\circ} 05.73^{\prime} \mathrm{W}$ & 1519 & 465 & 68 & 11 & \\
\hline $330,330 \mathrm{~A}$ & $50^{\circ} 55.19^{\prime} \mathrm{S} ; 46^{\circ} 53.00^{\prime} \mathrm{W}$ & 2626 & 576 & 47 & 47 & \\
\hline \multicolumn{7}{|l|}{ Leg 71} \\
\hline 511 & $51^{\circ} 00.28^{\prime} \mathrm{S} ; 46^{\circ} 58.30^{\prime} \mathrm{W}$ & 2589 & 632 & 127 & 60 & \multirow{4}{*}{$\begin{array}{l}\text { Jurassic } \\
\text { middle Eocene } \\
\text { early Oligocene } \\
\text { on basement } \\
\text { early Pliocene }\end{array}$} \\
\hline $512,512 \mathrm{~A}$ & $49^{\circ} 52.18^{\prime} \mathrm{S} ; 40^{\circ} 50.71^{\prime} \mathrm{W}$ & 1846 & 90 & 30 & & \\
\hline $513,513 \mathrm{~A}$ & $47^{\circ} 34.99^{\prime} \mathrm{S} ; 24^{\circ} 38.40^{\prime} \mathrm{W}$ & 4380 & 380 & 84 & 43 & \\
\hline 514 & $46^{\circ} 02.77^{\prime} \mathrm{S} ; 26^{\circ} 51.30^{\prime} \mathrm{W}$ & 4318 & 150 & 67 & & \\
\hline
\end{tabular}

The purpose of this study is to reconstruct the paleoenvironmental conditions in the region from the clay mineralogical and inorganic geochemical variations. Similar approaches have been used in the past to determine the paleoenvironmental evolution of the South Atlantic. Previous studies include the Cretaceous development of

${ }^{1}$ Ludwig, W. J., and Krasheninnikov, V. A., Init. Repts. DSDP, 71: Washington (U.S. Govt. Printing Office). the South Atlantic basins (Maillot, 1979; Robert et al., 1979; Maillot, 1980; Maillot and Robert, 1980), paleoenvironmental conditions during the deposition of the black shales (Chamley and Robert, in press), Cretaceous/Tertiary boundary events on the Atlantic margins (Chamley and Robert, 1979) and on the Rio Grande Rise (Robert, 1981), and Cenozoic changes in climates and currents in the South Atlantic (Maillot and Robert, 1980; Robert, 1980).

\section{METHODOLOGY}

\section{Clay Mineralogy}

The study involved the analysis of 543 samples. In each, the sediment fraction $<63 \mu \mathrm{m}$ was decalcified in $0.2 \mathrm{~N}$ hydrochloric acid. The excess acid was removed by repeated centrifugations followed by homogenization. The $<2 \mu \mathrm{m}$ fraction was separated by decantation (settling time based on Stokes' law), and then oriented aggregates were made on glass slides. Three X-ray diffractograms were made: (1) untreated sample, (2) glycolated sample, (3) sample heated for $2 \mathrm{hr}$. at $490^{\circ} \mathrm{C}$. A C.G.R. Theta 60 diffractometer (copper $\mathrm{K} \alpha$ radiation focused by a quartz curved crystal monochromator) was used at scan speeds of $1^{\circ} 2 \theta / \mathrm{min}$.; all instrument settings were kept constant. A receiving slit of $1.25 \mathrm{~mm}$ allowed a more precise resolution of poorly crystallized minerals.

The minerals recognized include chlorite, illite, irregular mixedlayer clays (chlorite-smectite and illite-smectite), kaolinite, smectite, and attapulgite (palygorskite). Associated minerals were quartz, feldspar, cristobalite, and clinoptilolite in variable abundance. The symbols used in Figures 3-10, Results, are shown in Figure 2.

Semiquantitative evaluations were based on the peak heights and areas. The height of the 001 illite peak (glycolated sample) was taken as a reference. Compared to this value, smectite, attapulgite, and irregular mixed-layer clays were corrected by multiplying their peak height by a factor of 1.5 to 2.5 , depending on their crystallinity, 


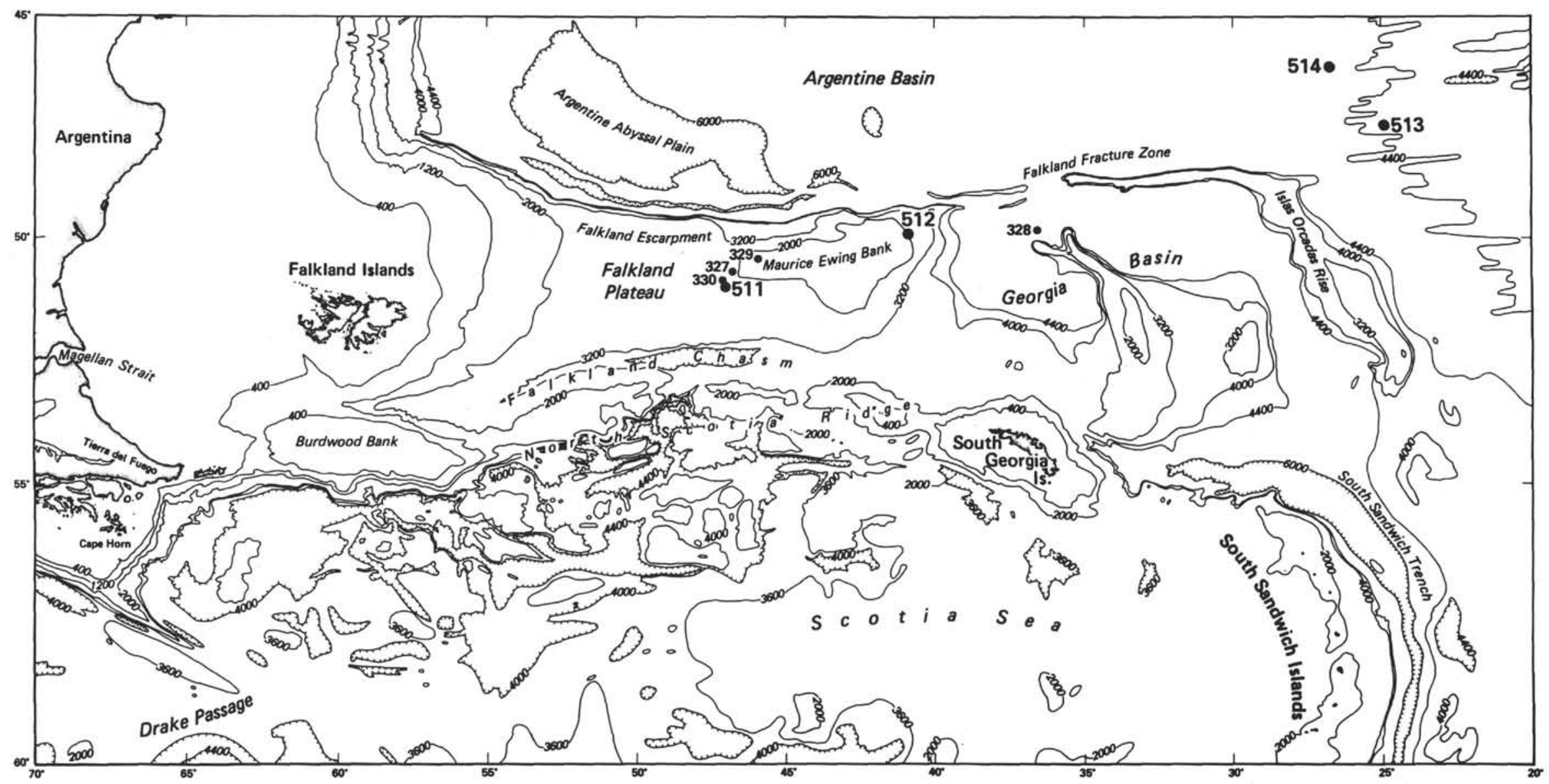

Figure 1. Site location map. 
Lithology

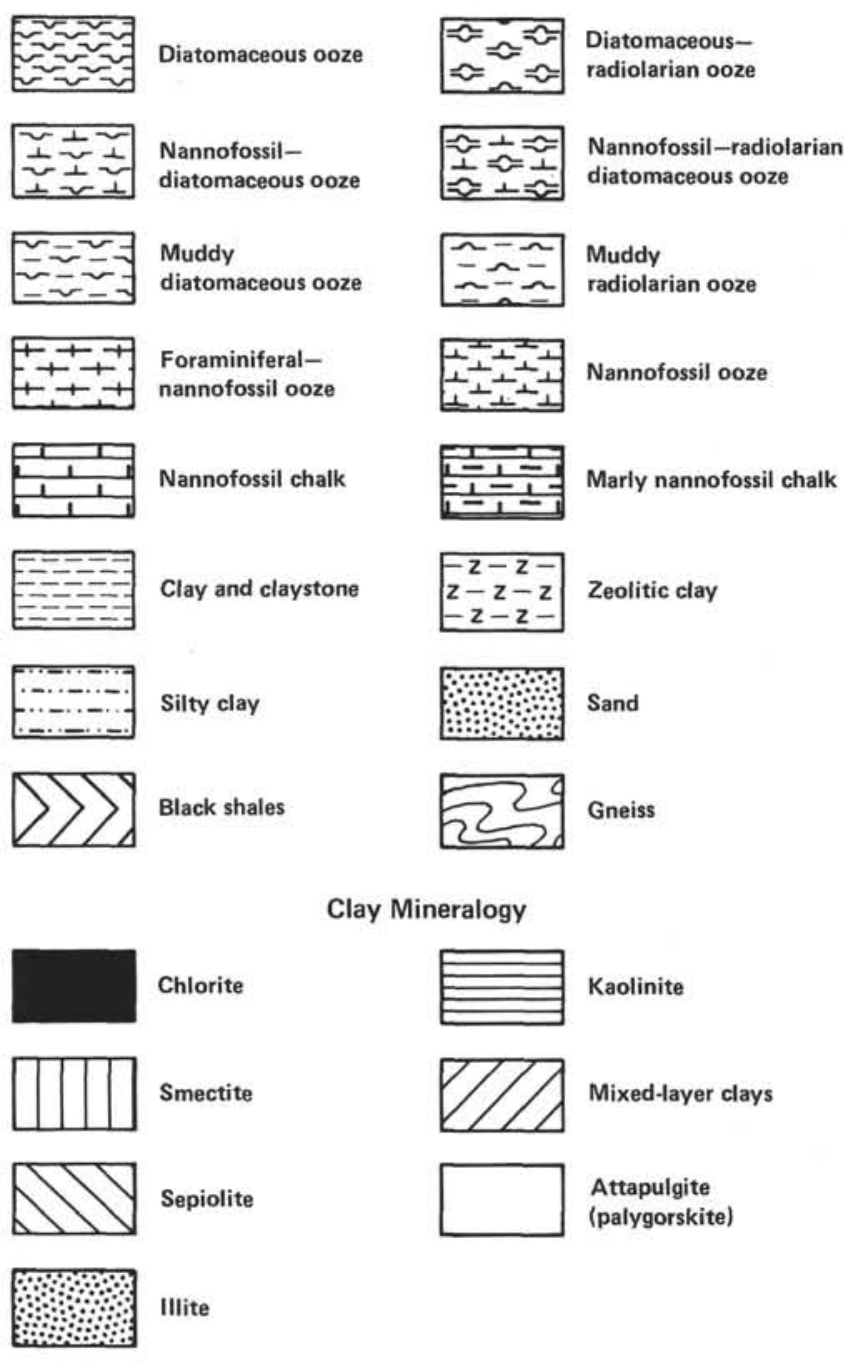

Associated Minerals

$\begin{array}{ll}\text { - Very rare } & \circ \text { Rare } \\ \text { - Abundant } & \circ \text { Very abundant }\end{array}$

Figure 2. Symbols used in Figures 3-10.

whereas well-crystallized kaolinite was corrected using a factor of 0.5 . Final data are given in percentages, the relative error being $\pm 5 \%$.

Illite crystallinity was measured by the width, at half height, of the 001 illite peak $(10 \AA)$, on the glycolated sample. The relative abundance of smectite versus illite $(\mathrm{S} / \mathrm{I}$ index) was obtained from the ratio of the 001 smectite $(18 \AA)$ and illite $(10 \AA)$ peaks, on the glycolated sample.

\section{Geochemistry}

The samples were dried at $105^{\circ} \mathrm{C}$, then ground and homogenized. Samples of $0.2 \mathrm{~g}$ were subjected to alkaline fusion, then dissolved in $\mathrm{HCl}$ and diluted to $100 \mathrm{ml}$. The treatment allowed gravimetric determination of $\mathrm{SiO}_{2}$ and spectrophotometric determination of $\mathrm{CaO}$, $\mathrm{MgO}, \mathrm{Al}_{2} \mathrm{O}_{3}$, and $\mathrm{Fe}_{2} \mathrm{O}_{3}$ (by atomic absorption). Another $2 \mathrm{~g}$ of each sample were subjected to fluoroperchloric treatment, then dissolved in $\mathrm{HCl}$ and diluted to $100 \mathrm{ml}$. The dilution was used for the colorimetric analysis of $\mathrm{TiO}_{2}$ and for the spectrophotometric analysis of $\mathrm{Na}_{2} \mathrm{O}$ and $\mathrm{K}_{2} \mathrm{O}$ (by emission) and of $\mathrm{Mn}, \mathrm{Zn}, \mathrm{Li}, \mathrm{Ni}, \mathrm{Cr}, \mathrm{Sr}, \mathrm{Cu}, \mathrm{Pb}, \mathrm{V}$, and $\mathrm{Cd}$ (by atomic absorption). The emission and atomic absorption apparatus was a Perkin Elmer type 503 spectrophotometer using the fol- lowing methods: (1) base solution for major elements, (2) complex synthetic solution for trace elements to which a solution of $5 \%$ lanthane in hydrochloric acid was added.

\section{GENERAL RESULTS}

\section{Clay Mineralogy}

In general, clay mineral assemblages from these sites show an absence of diagenetic modifications. (1) We observed no evidence of burial diagenesis: there is neither a progressive transition from smectite to mixed-layer clays and illite, nor a reduction in illite crystallinity with increased depth at these DSDP sites. (Compare Dunoyer's findings [1969] for the influence of burial diagenesis on clay minerals in deeper boreholes.) (2) Volcanic diagenesis is absent also: smectite abundance does not increase significantly toward the bottom of the holes which were nearer the spreading ridge when the sediments were deposited. Unfortunately, the base of the sedimentary section of Hole 513A, at the contact with the basalt, was not recovered. (3) Finally, there is no evidence of organic diagenesis: the black-shale environment shows the absence of in situ alteration of the clay minerals, in contrast to the similar reducing facies of Mediterranean Plio-Pleistocene sapropels (Sigl et al., 1978).

As there is no evidence for authigenesis of clay minerals in pelagic sediments in this part of the South Atlantic, they are considered to be essentially of detrital origin, formed in soils and by continental weathering as in the North Atlantic (Chamley, 1979) and in other parts of the South Atlantic (Robert et al., 1979; Robert, 1981).

In the Jurassic and Cretaceous sediments, kaolinite and large amounts of well-crystallized smectite, similar to the pedogenic smectite presently originating from African regions (Paquet, 1969), are present. These minerals point to the existence of a globally warm continental climate, in agreement with the data compiled by Frakes (1979). Clay mineral assemblages produced during long periods of constant, warm, climatic conditions (Millot, 1964; Chamley, 1979; Robert et al., 1979) are characterized by (1) chlorite, illite, and mixed-layer minerals, resulting principally from the direct erosion of rocks and from moderate continental weathering; (2) kaolinite, derived from soils developed in sloping and well-drained upstream areas; (3) smectite, coming mainly from deep soils in downstream areas of continental drainage basins, where relief is low and drainage poor; and (4) attapulgite (palygorskite), derived mainly from sediments of confined coastal basins.

In Cenozoic sediments, and especially since the late Eocene, the abundance of chlorite, illite, and mixedlayer clays increases progressively, following the general climatic cooling (Chamley, 1979) and the latitudinal zonation of climates and soils (Goldberg and Griffin, 1964; Pedro, 1968). The distribution of the clay minerals in the ocean is affected by several factors, the most important being the influence of oceanic water masses and circulation (Robert, 1980): in the Plio-Pleistocene sediments of the South Atlantic, intermediate water masses are enriched in illite and mixed-layer clays originating from temperate areas, North Atlantic Deep Water is 
marked by kaolinite from equatorial regions, and Antarctic Bottom Water contains an important proportion of smectite (Chamley, 1975).

\section{Geochemistry}

In oceanic sediments, $\mathrm{Fe}$ and $\mathrm{Mn}$ accumulations originate from materials that are detrital (fine or coarse fractions) hydrogenous, biogenic, and volcanogenic in origin, or by diagenetic processes (Elderfield, 1977). Despite these various origins, however, it seems that the relative abundances of $\mathrm{Fe}$ and $\mathrm{Mn}$ are significant for paleoenvironmental study (Turekian, 1965; Boström et al., 1972; Maillot, 1980; Maillot and Robert, 1980). For Krishnaswami (1976), Fe-Mn values higher than concentrations in a typical detrital shale are considered indicative of an authigenic influence in sediment formation. To show the influence of oceanic basalts and oxidizing currents, variations in the index

$\mathrm{Mn}^{*}=\log [\mathrm{Mn}$ sample/Mn shale $/ \mathrm{Fe}$ sample/Fe shale]

were studied, using the values of Mn shale and Fe shale given by Boström et al. (1976).

$\mathrm{Al}$ is principally related to the detritic minerals. An index proposed by Boström (Boström and Peterson, 1969; Boström, 1970), $\mathrm{D}^{*}=\mathrm{Al} / \mathrm{Al}+\mathrm{Fe}+\mathrm{Mn}$, associated with $\mathrm{Si}^{*}=\mathrm{SiO}_{2} / \mathrm{Al}_{2} \mathrm{O}_{3}$ permits recognition of detrital particles. $\mathrm{D}^{*}$ is close to 0.63 in typical terrigenous shales. A decrease of $D^{*}$ points to a less significant continental influence on sedimentation. Very high values of $\mathrm{D}^{*}$ correspond sometimes to abundant kaolinite particles. $\mathrm{Si}^{*}$ expresses the excess $\mathrm{Si}$ (i.e., Si not associated with $\mathrm{Al}$ ). This silica can be of volcanogenic (ashes), biogenic (diatoms, radiolarians), or detrital (quartz) origin.

The indexes $\mathrm{Mg}^{*}=\mathrm{MgO} / \mathrm{Al}_{2} \mathrm{O}_{3}$ and $\mathrm{Fe}^{*}=\mathrm{Fe}_{2} \mathrm{O}_{3} /$ $\mathrm{Al}_{2} \mathrm{O}_{3}$ permit interpretation of variations in $\mathrm{MgO}$ and $\mathrm{Fe}_{2} \mathrm{O}_{3}$ with respect to clay mineral abundances.

The index $\mathrm{Sr}^{*}=10^{3} \mathrm{Sr} / \mathrm{CaO}$ is generally related to the processes of carbonate dissolution (Maillot and Robert, 1980). When the variations related to burial diagenesis or to the primary composition of the carbonates are mathematically determined, $\mathrm{Sr}^{*}$ confirms the processes of carbonate dissolution as inferred by other methods (Maxwell et al., 1970; van Andel et al., 1977; Melguen et al., 1978a; Melguen et al., 1978b). Three types of dissolution appear:

1) Very high $\mathrm{Sr}^{*}$ : This type of dissolution is present in sediments rich in organic matter and depends on the intensity of the reduced sedimentary environment (Maillot and Robert, 1980).

2) High $\mathrm{Sr}^{*}$ : In open marine and oxidizing environments, this type of dissolution appears in the vicinity of the carbonate compensation depth (CCD). High $\mathrm{Sr}^{*}$ values result from the low solubility of $\mathrm{SrCO}_{3}$ compared with $\mathrm{CaCO}_{3}$ (Nekrassov, 1966) or from the partial adsorption of $\mathrm{Sr}$ by clay minerals (Bausch, 1968; Maillot, in press).

3) Low $\mathrm{Sr}^{*}$ : This type of dissolution occurs when the sediment has been deposited above the CCD (Maillot and Robert, 1980).

\section{FALKLAND PLATEAU}

Sites 330, 511, and 327

\section{Lithology (Figs. 3, 4, and 5)}

The base of the sedimentary section in the vicinity of Sites 330, 511, and 327 consists of Middle Jurassic sandstones and silty clays overlying a Precambrian gneiss (Barker, Dalziel, et al., 1977). From the Upper Jurassic to the Aptian/Albian boundary, a black shale facies is present; it consists of claystones at Site 327, claystones and limestones at Site 330, mudstones and nannofossil mudstones at Site 511. The Albian to Coniacian interval is represented by nannofossil claystones at Sites 327 and 330 and by alternating variegated claystones, nannofossil claystones, and muddy nannofossil chalks at Site 511. Then, zeolitic clays and claystones are present from the Santonian to the early Maestrichtian. Foraminiferal nannofossil oozes and calcareous oozes occur during Maestrichtian time and are replaced during Paleocene and early Eocene time by pelagic clay (Site 511), zeolitic clay, and clayey siliceous ooze (Site 327). Above that, a hiatus occurs at all sites. Early Oligocene sediments (Site 511) consist of muddy diatomaceous oozes and muddy nannofossil diatomaceous oozes. At the top of the sedimentary column, Quaternary sediments consist principally of gravelly sands, alternating with diatomaceous and foraminiferal oozes.

\section{Clay Mineralogy}

Several mineralogical units can be distinguished at these sites (Figs. 3, 4, and 5). In the Middle Jurassic and the lower section of the Upper Jurassic sediments of Hole 330 (Cores 15 to 11), kaolinite is the major clay mineral $(50 \pm 35 \%)$, accompanied by mixed-layer clays $(30 \pm 20 \%)$, illite $(25 \pm 20 \%)$, smectite $(30 \pm 30 \%)$, and chlorite $(5 \pm 5 \%)$; associated quartz occurs in small amounts. These clay mineral abundances are very similar to those of the mineral associations characteristic of the early Aptian of the Cape Basin and of the late Aptian-early Albian of the Angola Basin (Robert et al., 1979), where they marked a tectonic instability related to the opening of the basins.

In uppermost Jurassic, Neocomian, and Aptian sediments (Hole 330, Cores 10-3; Hole 511, Cores 70-60; Hole $327 \mathrm{~A}$, Cores 27-22), smectite increases ( $80 \pm$ $15 \%)$ relative to the underlying unit, whereas illite ( 15 $\pm 10 \%)$, mixed-layer clays $(5 \pm 5 \%)$, kaolinite $(2.5$ $\pm 2.5 \%)$, and chlorite $(2.5 \pm 2.5 \%)$ decrease. Small amounts of quartz, feldspar, and (locally) clinoptilolite and cristobalite occur. The transition from a clay mineral association where illite, mixed-layer clays, and kaolinite dominate toward one with abundant smectite indicates a decrease in tectonic activity, resulting in a diminution in erosional intensity and the development of flat coastal lowlands.

In Albian to Campanian sediments (Hole 330, Cores 2-1; Hole 511, Cores 58-25; Hole 327A, Cores 21-13), smectite $(97.5 \pm 2.5 \%)$ is the major mineral, accompanied by mixed-layer clays $(2.5 \pm 2.5 \%)$ and trace 
amounts of illite and (locally) chlorite. Quartz, feldspar, cristobalite, and clinoptilolite are also present. The great abundance of smectite suggests tectonic quiescence and generally subdued relief on the adjacent continental land masses. At Site 511, in the Albian-Turonian and the Coniacian-Santonian, two small increases in illite, mixed-layer clays, and kaolinite occur. These anomalies suggest increased erosion of altered rocks and of moderately and deeply weathered soils, pointing to tne occurrence of a local, moderate rejuvenation of the topography by tectonism.

The Maestrichtian-Paleocene sediments (Hole 511, Cores 25-22; Hole 327A, Cores 12-4) are rich in smectite $(97.5 \pm 2.5 \%$ of the clay fraction), accompanied by chlorite, illite, mixed-layer clays, kaolinite, and attapulgite $(2.5 \pm 2.5 \%)$. A short hiatus near the Cretaceous/ Tertiary boundary occurs during this interval in Hole 511. Sediments corresponding in age to this hiatus were recovered from Hole 327A, however, and are characterized by a small increase in primary minerals $(2.5 \pm$ $2.5 \%)$, mixed-layer clays $(10 \pm 10 \%)$, and kaolinite (trace amounts) both above and below the Cretaceous/ Tertiary boundary that seems to result from a moderate, marginal tectonic rejuvenation (Chamley and Robert, 1979).

In Eocene sediments (Hole 330A, Core 1; Hole 511, Cores 21-17; Hole 327A, Core 2), smectite prevails in the clay fraction $(90 \pm 5 \%)$ and is accompanied by mixed-layer clays $(5 \pm 5 \%)$, illite $(2.5 \pm 2.5 \%)$, chlorite, kaolinite, and attapulgite (trace amounts). This clay mineral association indicates a period of tectonic quiescence with a globally warm climate and major seasonal changes in humidity. However, in Hole $330 \mathrm{~A}$, Core 1 displays a short period in which illite $(7.5 \pm 2.5 \%)$ and mixed-layer clays $(15 \pm 5 \%)$ increase.

Early Oligocene sediments (Hole 511, Cores 17-1) are characterized by a progressive increase in chlorite ( 7.5 $\pm 2.5 \%)$, illite $(15 \pm 10 \%)$, and mixed-layer clays $(2.5$ $\pm 2.5 \%$ ) that is related to progressive climatic cooling (Chamley, 1979). Smectite decreases $(65 \pm 15 \%)$; kaolinite $(2.5 \pm 2.5 \%)$ and attapulgite (trace amounts) are also present.

In Pleistocene sediments (Hole 327A, Core 1), smectite remains the major mineral $(85 \pm 5 \%)$ and is accompanied by chlorite and illite $(2.5 \pm 2.5 \%)$, mixed-layer clays $(7.5 \pm 2.5 \%)$, and trace amounts of kaolinite.

\section{Geochemistry (Figs. 3, 4, and 5; Tables 1, 2, and 3)}

In Middle Jurassic sediments (Hole 330, Core 15), D* is very high ( 0.81$)$, pointing to a strong detrital influence or to the presence of abundant kaolinite. $\mathrm{Mn}^{*}$ has a negative value $(-0.58)$ that indicates a reducing environment. Organic acids completely dissolved the carbonates: $\mathrm{Sr}^{*}$ is very high (31.24) and marks the retention of the strontium in the sediments at the time of the dissolution. The low values of $\mathrm{Mg}^{*}(0.03)$ and $\mathrm{Fe}^{*}(0.19)$ are characteristic of a nearby continental influence and of a detrital clay mineral assemblage.

In Upper Jurassic sediments (Hole 330, Cores 14-8), a high value of $\mathrm{D}^{*}(0.53-0.67)$ suggests a high detrital input from the continent. The slight decrease in $\mathrm{D}^{*}$ in the early Upper Jurassic (Fig. 4) occurs contemporaneously with the development of a shallow marine environment on the Maurice Ewing Bank. Mn* remains negative, indicating that organic components are important and that the environment is a reducing one. $\mathrm{Sr}^{*}$ is related to the dissolution induced by presence of organic matter.

In Upper Jurassic to Aptian sediments, $\mathrm{Mn}^{*}$ is always negative, indicating a reducing environment. $\mathrm{Sr}^{*}$ decreases $(16.56-6.46)$ and is very close to the values recorded in marine carbonates. The sediment is characterized by indications of a continental detrital supply and by marine biogenic deposits.

At the end of the late Aptian (Hole 327) or at the beginning of the Albian (Holes 330 and 511), an open marine environment appeared. The values of $\mathrm{Sr}^{*}(1.20-$ $2.04)$ become typical of marine carbonates. $D^{*}(0.55-$ $0.61)$ diminishes with decreasing continental influence and $\mathrm{Mn}^{*}$ becomes positive $(0.56-1.12)$ in response to the presence of an oxidizing environment which persisted until Coniacian-Santonian time.

In Coniacian-Santonian sediments, $\mathrm{D}^{*}$ is again high (0.66), pointing to an increase in the detrital influence, whereas $\mathrm{Mn}^{*}$ fluctuates between positive and negative values. This alternation could correspond to the period during which the site was near the boundary between an oxidized upper water mass and a reduced deep water mass.

During the Santonian, Campanian, and Maestrichtian, $\mathrm{D}^{*}$ decreases slightly $(0.62)$ and $\mathrm{Mn}^{*}$ remains negative $(-0.27)$, indicating the persistent presence of a reducing environment that is probably related to the location of the site in deep (or intermediate) water masses. During the Maestrichtian, $\mathrm{Mn}^{*}$ becomes positive (0.610.78 ). The change is possibly related to variations in the intensity of ocean currents or to fluctuations in the sea level.

In the Paleocene, $\mathrm{D}^{*}$ decreases slightly $(0.58)$ whereas $\mathrm{Mn}^{*}$ becomes negative again $(-0.51)$. A reducing environment was probably induced by the influence of deep or intermediate water masses. The same environment persists until the early Oligocene.

\section{Sites 329, 512}

\section{Lithology (Figs. 6, 7)}

The base of the sedimentary section, from the upper Paleocene to the Oligocene/Miocene boundary (Site 329 ) and during middle Eocene time (Site 512), consists of nannofossil chalks and oozes. Above that, lower to upper Miocene (Site 329) and middle Miocene (Site 512) sediments consist of siliceous nannofossil oozes and upper Miocene to Pleistocene sediments of diatomaceous oozes and sand.

\section{Clay Mineralogy (Figs. 6-7)}

In upper Paleocene and lower and middle Eocene sediments (Hole 329, Cores 33 and 32; Hole 512, Cores 19-6 and Hole 512A, Core 2), smectite represents 95 $\pm 5 \%$ of the clay fraction. Chlorite, illite, mixed-layer clays, kaolinite, and attapulgite are also present ( $2.5 \pm$ $2.5 \%$ ). These clay mineral abundances are very close to 


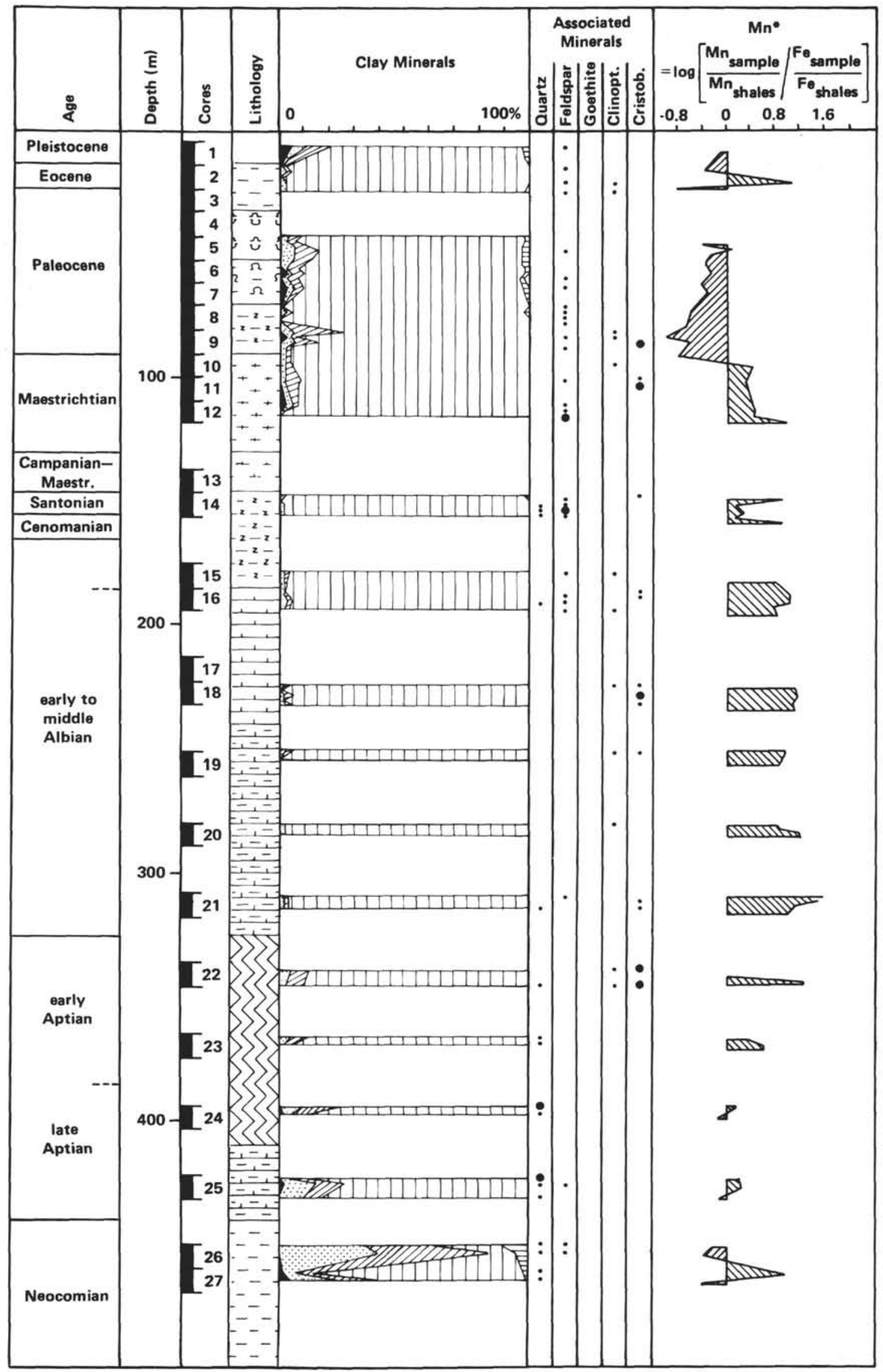

Figure 3. Hole 327A results. 
PALEOENVIRONMENTAL SIGNIFICANCE OF CLAY MINERAL DATA

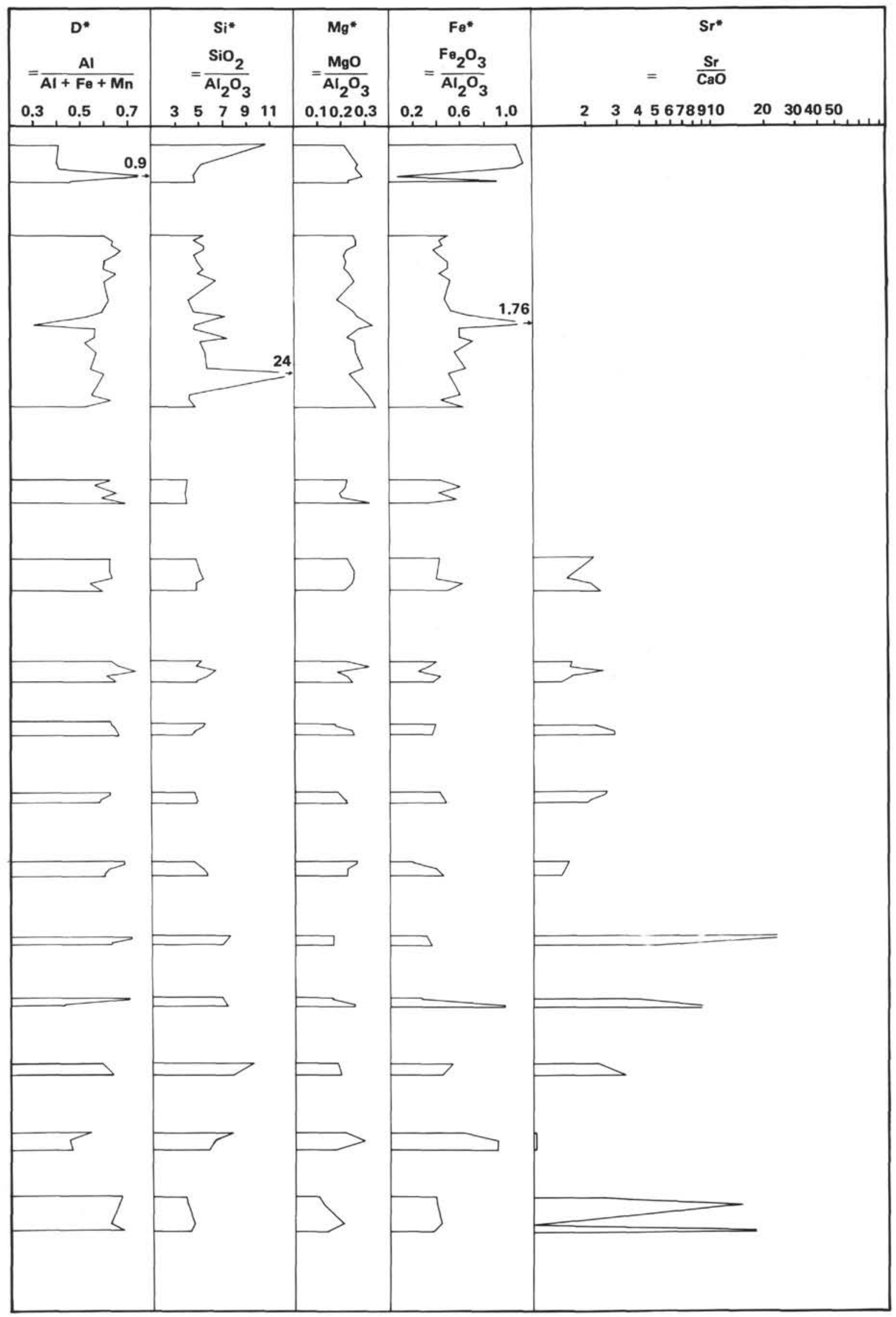

Figure 3. (Continued). 


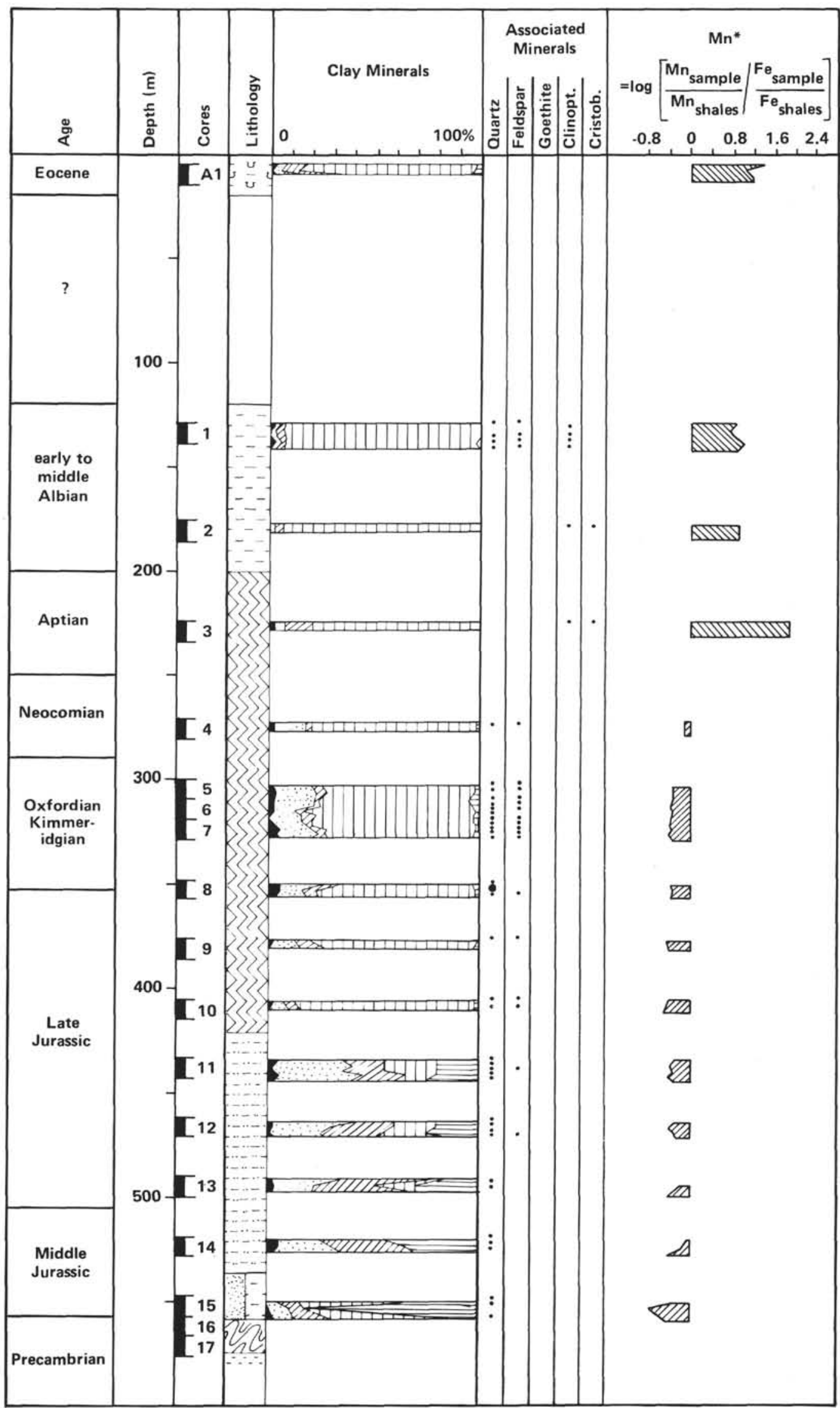

Figure 4. Holes 330 and $330 \mathrm{~A}$ results. 
PALEOENVIRONMENTAL SIGNIFICANCE OF CLAY MINERAL DATA

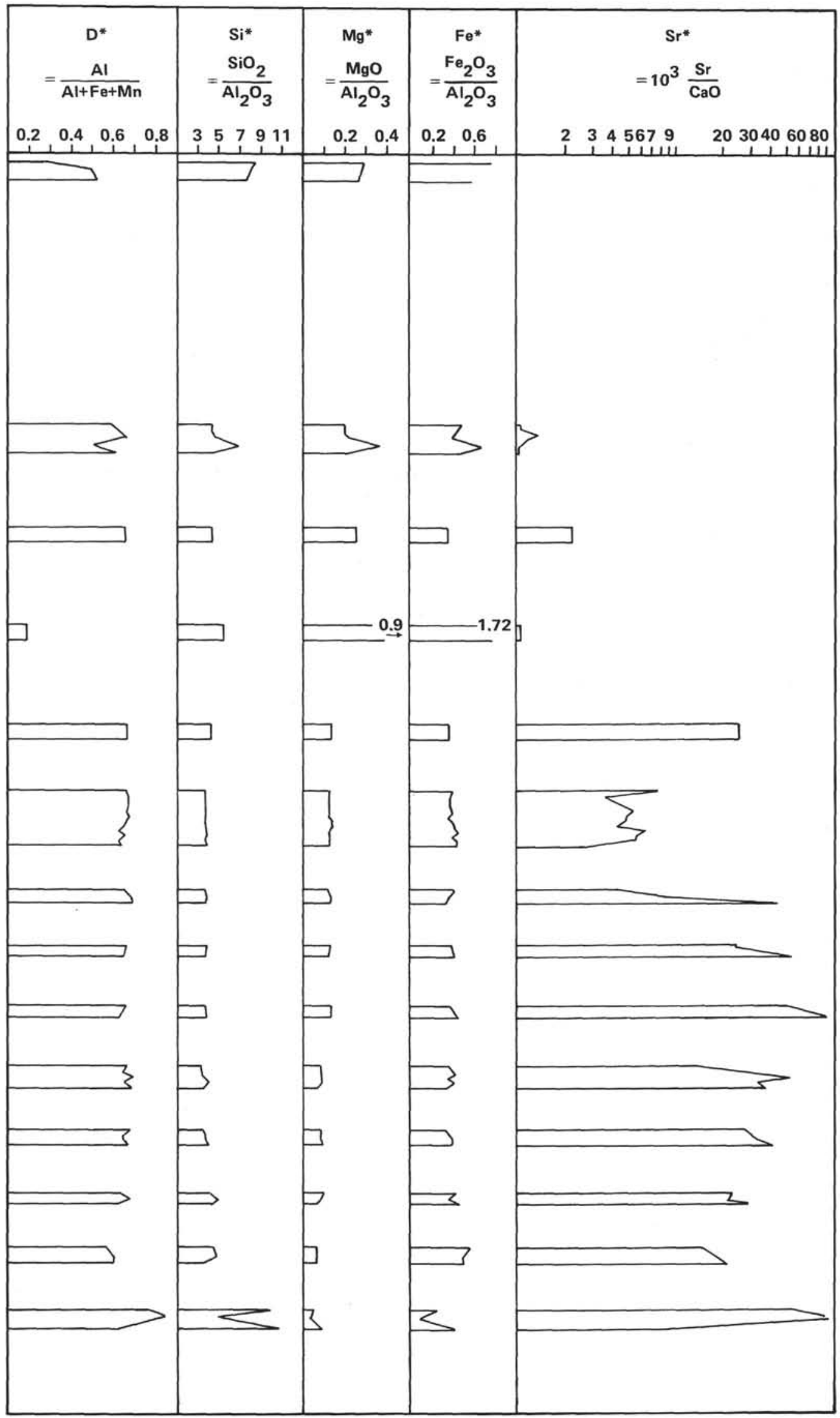

Figure 4. (Continued). 


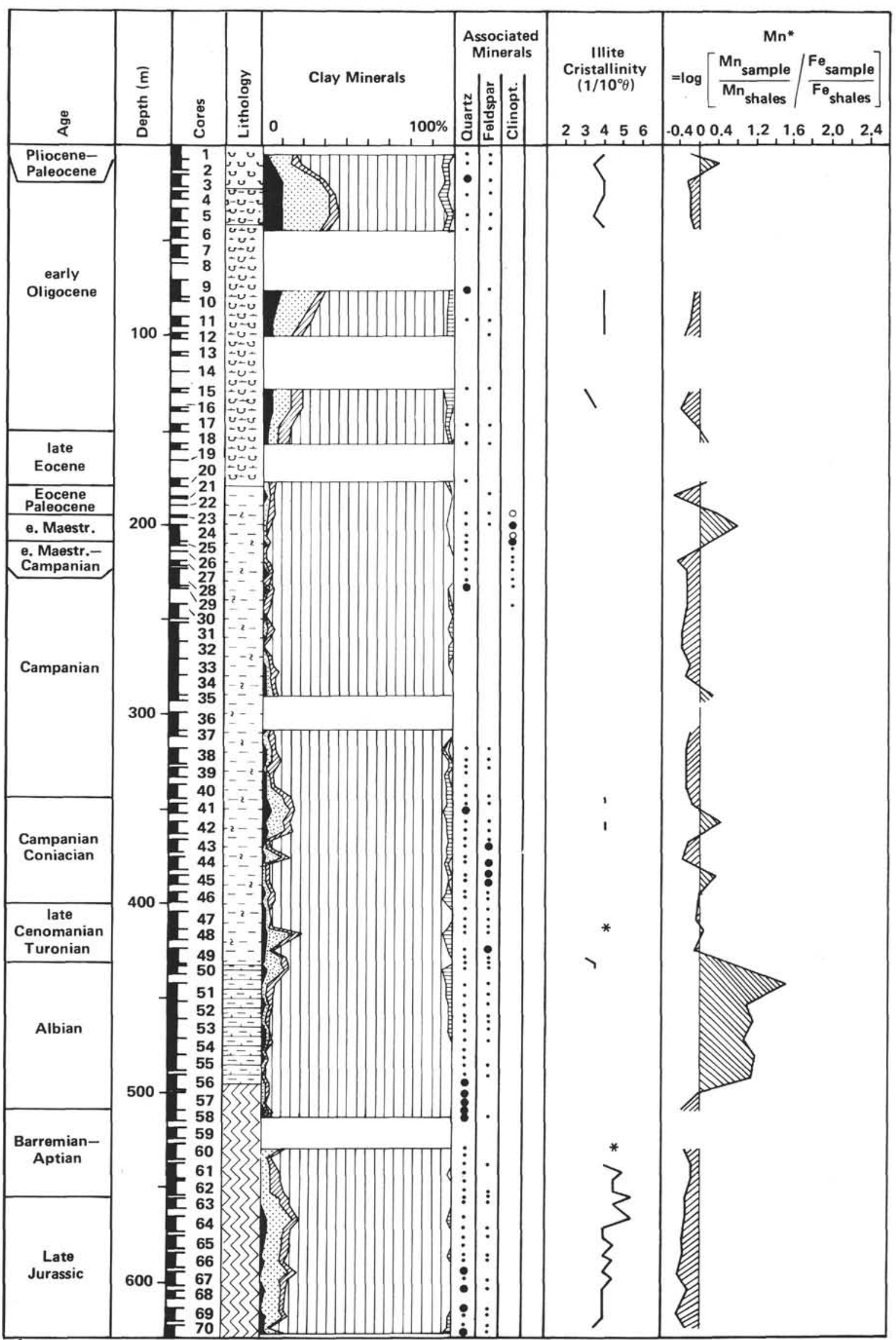

Figure 5. Hole 511 results. 
PALEOENVIRONMENTAL SIGNIFICANCE OF CLAY MINERAL DATA

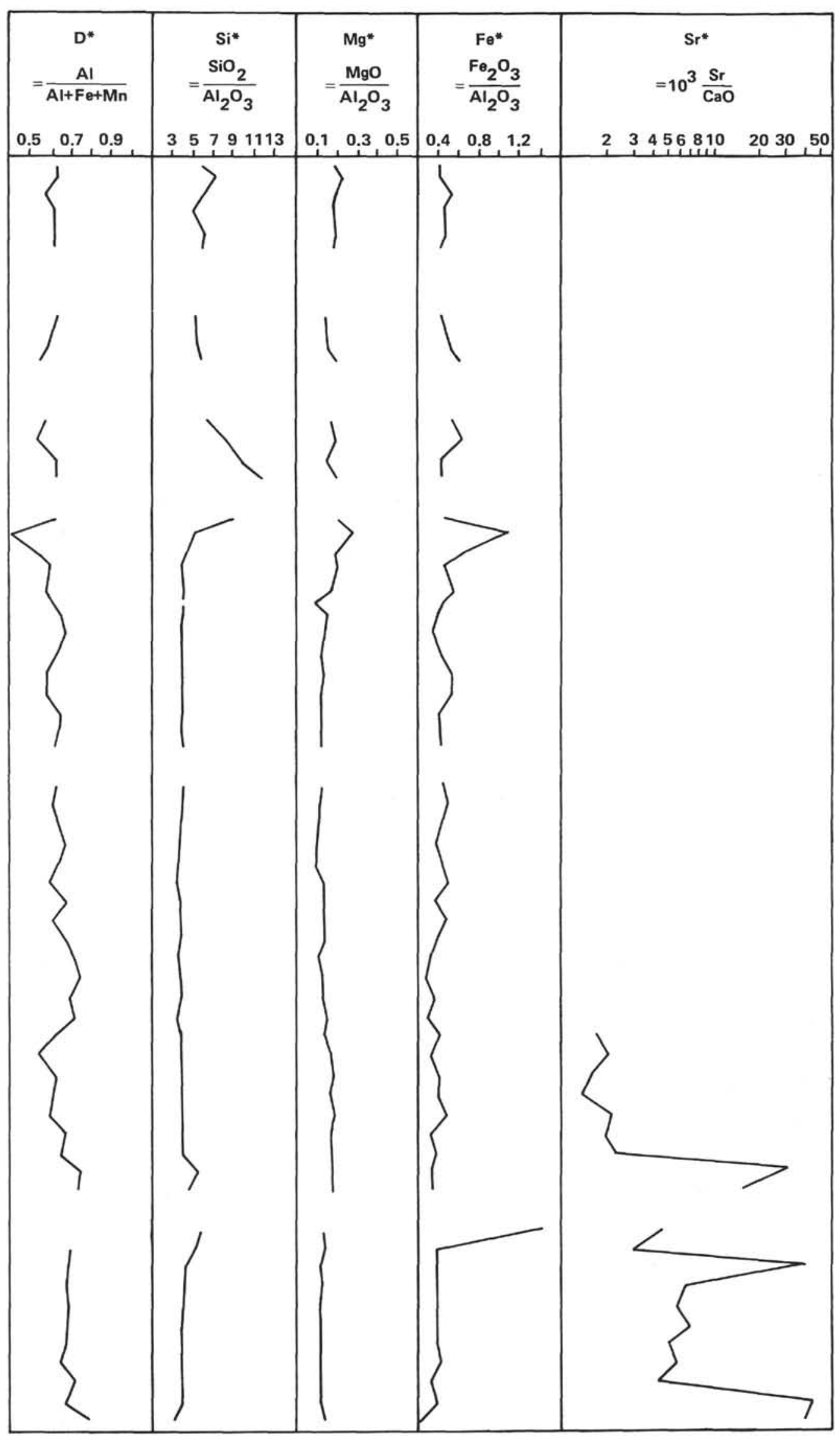

Figure 5. (Continued). 
Table 1. Hole 511 geochemical results.

\begin{tabular}{|c|c|c|c|c|c|c|c|c|c|c|c|c|c|c|c|c|c|c|c|c|}
\hline $\begin{array}{l}\text { Core/Section } \\
\text { (level in } \mathrm{cm} \text { ) }\end{array}$ & $\underset{(\%)}{\mathrm{SiO}_{2}}$ & $\underset{(\%)}{\mathrm{Al}_{2} \mathrm{O}_{3}}$ & $\begin{array}{l}\mathrm{CaO} \\
(\%)\end{array}$ & $\begin{array}{c}\mathrm{MgO} \\
(\%)\end{array}$ & $\underset{(\%)}{\mathrm{Na}_{2} \mathrm{O}}$ & $\begin{array}{l}\mathrm{K}_{2} \mathrm{O} \\
(\%)\end{array}$ & $\underset{(\%)}{\mathrm{TiO}_{2}}$ & $\begin{array}{l}\mathrm{P}_{2} \mathrm{O}_{5} \\
(\%)\end{array}$ & $\begin{array}{c}\mathrm{Fe}_{2} \mathrm{O}_{3} \\
(\%)\end{array}$ & $\begin{array}{c}\mathrm{Mn} \\
(\mathrm{ppm})\end{array}$ & $\underset{(\mathrm{ppm})}{\mathrm{Zn}}$ & $\underset{(\mathrm{ppm})}{\mathrm{Li}}$ & $\begin{array}{c}\mathrm{Ni} \\
(\mathrm{ppm})\end{array}$ & $\underset{(\mathrm{ppm})}{\mathrm{Cr}}$ & $\underset{(\mathrm{ppm})}{\mathrm{Sr}}$ & $\begin{array}{c}\text { Co } \\
(\mathrm{ppm})\end{array}$ & $\underset{(\mathrm{ppm})}{\mathrm{Cu}}$ & $\begin{array}{c}\mathrm{Pb} \\
\text { (ppm) }\end{array}$ & $\underset{(\mathrm{ppm})}{\mathrm{V}}$ & $\underset{\text { (ppm) }}{\mathrm{Cd}}$ \\
\hline $1-4,15$ & 58.15 & 10.75 & 0.84 & 2.13 & 3.61 & 2.67 & 0.67 & n.d. & 4.50 & 279 & 142 & 67 & 39 & 58 & 147 & 1 & 35 & 17 & 95 & 1 \\
\hline $2-3,10$ & 60.40 & 8.27 & 1.26 & 1.89 & 3.98 & 2.11 & 0.54 & n.d. & 3.36 & 779 & 105 & 49 & 18 & 45 & 168 & 1 & 19 & 61 & 77 & 1 \\
\hline $3-4,8$ & 55.60 & 8.77 & 6.05 & 1.75 & 3.40 & 2.53 & 0.49 & n.d. & 4.86 & 268 & 258 & 46 & 23 & 62 & 300 & 5 & 28 & 32 & 89 & 2 \\
\hline $4-2,98$ & 57.95 & 11.16 & 4.44 & 1.96 & 2.73 & 2.67 & 0.60 & n.d. & 5.08 & 321 & 116 & 43 & 20 & 56 & 268 & 6 & 23 & 31 & 95 & 2 \\
\hline $5-3,90$ & 62.65 & 10.10 & 2.59 & 2.02 & 4.70 & 2.54 & 0.55 & n.d. & 4.72 & 289 & 111 & 35 & 7 & 48 & 205 & 2 & 16 & 47 & 71 & 1 \\
\hline $6-2,50$ & 56.35 & 9.45 & 3.64 & 1.77 & 3.67 & 2.47 & 0.47 & n.d. & 3.93 & 289 & 105 & 34 & 8 & 44 & 253 & 1 & 24 & 35 & 84 & 3 \\
\hline $9-5,50$ & 49.25 & 9.33 & 4.27 & 1.38 & 2.70 & 2.20 & 0.55 & n.d. & 4.00 & 289 & 105 & 30 & 8 & 48 & 284 & 3 & 16 & 39 & 74 & 2 \\
\hline $11-3,10$ & 56.45 & 10.20 & 3.81 & 1.59 & 2.59 & 2.45 & 0.59 & n.d. & 5.36 & 316 & 111 & 29 & 17 & 49 & 242 & 5 & 20 & 40 & 92 & 3 \\
\hline $12-2,80$ & 57.35 & 10.04 & 1.75 & 2.08 & 2.39 & 2.53 & 0.63 & n.d. & 6.29 & 279 & 147 & 41 & 31 & 77 & 179 & 18 & 20 & 43 & 116 & 2 \\
\hline $15-1,80$ & 57.10 & 8.90 & 4.20 & 1.62 & 2.93 & 2.11 & 0.56 & n.d. & 4.90 & 279 & 121 & 38 & 25 & 51 & 274 & 5 & 22 & 34 & 74 & 2 \\
\hline $16-1,80$ & 65.05 & 7.79 & 0.42 & 1.56 & 2.56 & 1.93 & 0.48 & n.d. & 5.04 & 200 & 121 & 39 & 14 & 63 & 132 & 1 & 25 & 33 & 92 & 1 \\
\hline $17-2,50$ & 61.30 & 6.35 & 5.25 & 1.04 & 2.56 & 1.31 & 0.44 & n.d. & 2.82 & 242 & 105 & 31 & 3 & 38 & 295 & 0 & 13 & 29 & 51 & 1 \\
\hline $18-1,60$ & 59.90 & 5.14 & 8.15 & 1.10 & 2.95 & 1.08 & 0.33 & n.d. & 2.25 & 300 & 158 & 25 & 2 & 33 & 358 & 1 & 7 & 39 & 42 & 2 \\
\hline $20-2,58$ & 57.20 & 6.35 & 9.58 & 1.31 & 2.12 & 1.20 & 0.43 & n.d. & 2.72 & 353 & 116 & 30 & 14 & 37 & 379 & 2 & 23 & 34 & 53 & 2 \\
\hline $21-1,51$ & 56.05 & 11.04 & 1.05 & 3.22 & 2.31 & 2.32 & 0.67 & n.d. & 12.15 & 300 & 300 & 88 & 178 & 101 & 211 & 26 & 27 & 65 & 103 & 2 \\
\hline $23-1,34$ & 32.10 & 7.44 & 23.61 & 1.46 & 1.82 & 1.84 & 0.33 & n.d. & 4.68 & 1089 & 168 & 53 & 42 & 40 & 711 & 8 & 21 & 39 & 66 & 2 \\
\hline $24-1,43$ & 21.75 & 5.53 & 33.58 & 1.17 & 1.35 & 1.17 & 0.27 & n.d. & 2.54 & 1495 & 142 & 29 & 31 & 29 & 1211 & 12 & 37 & 48 & 75 & 3 \\
\hline $26, \mathrm{CC}$ & 58.05 & 13.99 & 0.45 & 2.51 & 2.22 & 2.79 & 0.72 & n.d. & 7.65 & 579 & 163 & 61 & 38 & 63 & 179 & 10 & 28 & 45 & 150 & 1 \\
\hline $27-1,42$ & 59.50 & 14.64 & 0.28 & 2.24 & 2.56 & 2.73 & 0.72 & n.d. & 6.68 & 211 & 153 & 46 & 31 & 57 & 211 & 12 & 11 & 59 & 116 & 1 \\
\hline $28-2,50$ & 60.15 & 15.00 & 0.28 & 2.46 & 2.22 & 2.67 & 0.72 & n.d. & 5.93 & 295 & 168 & 49 & 32 & 61 & 216 & 9 & 91 & 55 & 211 & 2 \\
\hline $29-1,13$ & 54.85 & 14.29 & 4.90 & 2.09 & 2.21 & 2.17 & 0.73 & n.d. & 5.04 & 258 & 174 & 36 & 25 & 51 & 263 & 11 & 30 & 55 & 137 & 3 \\
\hline $30-2,80$ & 55.55 & 14.17 & 4.16 & 1.85 & 2.02 & 2.54 & 0.65 & n.d. & 6.15 & 321 & 158 & 32 & 21 & 49 & 274 & 9 & 30 & 50 & 119 & 1 \\
\hline $31-3,130$ & 53.95 & 13.65 & 4.51 & 1.95 & 2.22 & 2.36 & 0.63 & n.d. & 7.36 & 311 & 163 & 31 & 28 & 56 & 263 & 13 & 27 & 61 & 124 & 1 \\
\hline $32-3,78$ & 56.75 & 14.76 & 2.20 & 1.95 & 1.85 & 2.18 & 0.68 & n.d. & 6.79 & 263 & 163 & 33 & 18 & 55 & 221 & 8 & 31 & 52 & 130 & 2 \\
\hline $33-3,130$ & 53.25 & 14.29 & 5.63 & 1.83 & 1.85 & 2.02 & 0.69 & n.d. & 5.68 & 332 & 200 & 34 & 21 & 52 & 268 & 8 & 35 & 47 & 118 & 2 \\
\hline $34-1,36$ & 55.15 & 14.76 & 3.88 & 1.92 & 2.12 & 2.15 & 0.72 & n.d. & 6.08 & 289 & 163 & 36 & 26 & 61 & 253 & 12 & 38 & 43 & 141 & 1 \\
\hline $35-1,50$ & 53.30 & 13.58 & 6.72 & 1.70 & 1.85 & 2.02 & 0.68 & n.d. & 5.83 & 995 & 211 & 32 & 32 & 54 & 347 & 15 & 34 & 61 & 124 & 2 \\
\hline $37-1,78$ & 52.20 & 13.46 & 6.86 & 1.69 & 1.99 & 2.06 & 0.68 & n.d. & 5.86 & 353 & 142 & 34 & 26 & 53 & 379 & 13 & 35 & 47 & 126 & 2 \\
\hline $38-2,60$ & 52.50 & 14.02 & 4.83 & 1.63 & 1.75 & 2.41 & 0.69 & n.d. & 6.83 & 326 & 163 & 40 & 38 & 58 & 274 & 17 & 34 & 49 & 128 & 3 \\
\hline $39-1,125$ & 53.60 & 14.78 & 4.69 & 1.66 & 1.97 & 2.53 & 0.75 & n.d. & 6.08 & 321 & 163 & 46 & 31 & 59 & 263 & 16 & 39 & 41 & 137 & 2 \\
\hline $40-2,14$ & 57.35 & 16.53 & 1.22 & 1.61 & 1.74 & 2.82 & 0.73 & n.d. & 6.00 & 284 & 211 & 68 & 32 & 65 & 189 & 16 & 46 & 34 & 157 & 2 \\
\hline $41-1,80$ & 51.80 & 15.00 & 6.02 & 1.49 & 1.75 & 2.95 & 0.71 & n.d. & 6.33 & 400 & 226 & 76 & 32 & 58 & 263 & 19 & 18 & 47 & 104 & 2 \\
\hline $42-1,92$ & 47.95 & 15.00 & 5.14 & 1.97 & 1.79 & 2.60 & 0.57 & n.d. & 7.51 & 1884 & 174 & 84 & 37 & 45 & 211 & 15 & 5 & 32 & 118 & 1 \\
\hline $43-1,30$ & 53.85 & 15.23 & 3.22 & 2.20 & 2.17 & 2.24 & 0.70 & n.d. & 5.40 & 284 & 174 & 42 & 39 & 37 & 205 & 20 & 13 & 38 & 103 & 1 \\
\hline $44-1,52$ & 56.25 & 15.71 & 0.28 & 2.16 & 1.98 & 2.99 & 0.52 & n.d. & 7.48 & 295 & 132 & 45 & 38 & 47 & 132 & 22 & 25 & 42 & 108 & 0 \\
\hline $45-1,94$ & 51.00 & 14.05 & 6.37 & 1.99 & 2.00 & 2.45 & 0.63 & n.d. & 5.11 & 1042 & 179 & 38 & 25 & 42 & 247 & 17 & 18 & 35 & 108 & 1 \\
\hline $46-1,50$ & 55.50 & 16.41 & 3.11 & 1.78 & 1.97 & 2.71 & 0.71 & n.d. & 5.04 & 500 & 195 & 69 & 29 & 48 & 179 & 19 & 32 & 35 & 117 & 3 \\
\hline $47-1,38$ & 57.10 & 15.71 & 3.50 & 2.10 & 2.16 & 2.60 & 0.63 & n.d. & 4.04 & 347 & 168 & 39 & 51 & 45 & 226 & 38 & 41 & 48 & 92 & 1 \\
\hline $48-1,38$ & 54.65 & 14.47 & 5.49 & 1.91 & 1.87 & 2.62 & 0.53 & n.d. & 4.86 & 542 & 153 & 49 & 44 & 43 & 226 & 17 & 31 & 41 & 68 & 1 \\
\hline $49-2,23$ & 57.10 & 16.77 & 0.98 & 2.59 & 2.18 & 2.62 & 0.70 & n.d. & 4.79 & 400 & 158 & 49 & 48 & 43 & 189 & 39 & 46 & 51 & 137 & 1 \\
\hline $50-1,23$ & 44.55 & 12.20 & 12.17 & 1.74 & 1.87 & 3.30 & 0.42 & n.d. & 4.97 & 2521 & 168 & 45 & 35 & 19 & 226 & 28 & 2 & 48 & 68 & 2 \\
\hline $51-1,130$ & 40.30 & 10.92 & 14.52 & 1.88 & 1.85 & 2.17 & 0.38 & n.d. & 3.57 & $2.37 \%$ & 147 & 52 & 78 & 12 & 342 & 30 & 101 & 107 & 55 & 2 \\
\hline $52-2,100$ & 37.00 & 9.93 & 17.84 & 1.82 & 1.56 & 1.79 & 0.38 & n.d. & 4.11 & 3484 & 126 & 67 & 53 & 17 & 295 & 27 & 31 & 55 & 53 & 2 \\
\hline $53-1,130$ & 36.45 & 9.48 & 18.43 & 1.60 & 1.57 & 2.11 & 0.37 & n.d. & 3.82 & 4868 & 105 & 49 & 43 & 16 & 289 & 24 & 23 & 47 & 39 & 2 \\
\hline $54-2,25$ & 42.55 & 10.72 & 12.91 & 2.00 & 1.79 & 1.99 & 0.49 & n.d. & 5.11 & 3447 & 142 & 53 & 53 & 19 & 295 & 28 & 13 & 47 & 42 & 3 \\
\hline $55-2,42$ & 45.80 & 11.99 & 12.17 & 2.04 & 1.85 & 2.11 & 0.35 & n.d. & 3.79 & 5021 & 147 & 68 & 47 & 20 & 258 & 23 & 21 & 37 & 45 & 2 \\
\hline $56-2,71$ & 47.45 & 12.07 & 10.77 & 2.03 & 1.72 & 1.51 & 0.53 & n.d. & 4.47 & 5190 & 105 & 28 & 209 & 18 & 263 & 41 & 33 & 32 & 53 & 1 \\
\hline $57-2,35$ & 67.25 & 12.02 & 0.52 & 2.16 & 1.87 & 1.76 & 0.34 & n.d. & 3.15 & 274 & 379 & 58 & 272 & 35 & 168 & 28 & 86 & 58 & 121 & 1 \\
\hline $58-1,38$ & 63.20 & 13.93 & 0.52 & 2.45 & 2.01 & 1.26 & 0.68 & n.d. & 3.90 & 137 & 300 & 48 & 336 & 23 & 184 & 22 & 13 & 25 & 66 & 0 \\
\hline $60-3,18$ & 35.40 & 6.31 & 14.76 & 0.79 & 1.08 & 1.72 & 0.29 & n.d. & 8.94 & 384 & 179 & 24 & 331 & 29 & 226 & 31 & 64 & 39 & 329 & 6 \\
\hline $61-2,80$ & 54.15 & 10.72 & 5.60 & 1.48 & 1.57 & 2.39 & 0.37 & n.d. & 3.68 & 226 & 337 & 42 & 86 & 53 & 168 & 4 & 36 & 32 & 668 & 6 \\
\hline $62-2,80$ & 58.60 & 13.62 & 0.38 & 1.65 & 1.60 & 3.25 & 0.63 & n.d. & 4.86 & 316 & 205 & 42 & 37 & 152 & 153 & 5 & 42 & 20 & 208 & 1 \\
\hline $63-1,30$ & 54.75 & 13.63 & 2.41 & 1.76 & 1.71 & 3.46 & 0.52 & n.d. & 5.15 & 242 & 563 & 44 & 72 & 62 & 163 & 4 & 29 & 38 & 621 & 5 \\
\hline $64-2,75$ & 54.40 & 14.05 & 2.55 & 1.58 & 1.48 & 4.19 & 0.56 & n.d. & 4.90 & 232 & 511 & 43 & 53 & 68 & 153 & 6 & 32 & 38 & 592 & 5 \\
\hline $65-2,14$ & 52.80 & 14.05 & 2.24 & 1.67 & 1.58 & 3.73 & 0.54 & n.d. & 5.15 & 205 & 379 & 43 & 99 & 63 & 158 & 7 & 36 & 42 & 568 & 4 \\
\hline $66-2,32$ & 52.80 & 13.58 & 3.15 & 1.58 & 1.57 & 3.46 & 0.48 & n.d. & 4.93 & 205 & 426 & 40 & 130 & 68 & 168 & 9 & 46 & 40 & 713 & 6 \\
\hline $67-1,90$ & 51.55 & 13.46 & 2.94 & 1.58 & 1.58 & 3.46 & 0.52 & n.d. & 5.61 & 195 & 532 & 41 & 126 & 61 & 174 & 9 & 49 & 47 & 553 & 4 \\
\hline $68-1,23$ & 53.20 & 13.82 & 3.99 & 1.62 & 1.57 & 3.30 & 0.54 & n.d. & 4.25 & 221 & 253 & 41 & 64 & 82 & 174 & 3 & 34 & 49 & 482 & 3 \\
\hline $69-2,34$ & 57.60 & 15.12 & 0.31 & 1.85 & 1.72 & 3.46 & 0.60 & n.d. & 5.75 & 184 & 200 & 49 & 47 & 99 & 137 & 3 & 31 & 40 & 221 & 1 \\
\hline $70-2,68$ & 54.60 & 18.30 & 0.63 & 2.63 & 2.44 & 1.78 & 0.44 & n.d. & 3.65 & 179 & 121 & 45 & 16 & 85 & 247 & 4 & 11 & 44 & 234 & 1 \\
\hline
\end{tabular}

Note: n.d. $=$ no data.

those of the mineral assembly represented in Holes $327 \mathrm{~A}, 330$, and 511 .

In Oligocene sediments (Hole 329, Cores 31-28), smectite abundance decreases slightly $(85 \pm 10 \%)$, as observed at Site 511. Associated minerals include chlorite (trace amounts), illite $(5 \pm 5 \%)$, mixed-layer clays $(2.5 \pm 2.5 \%)$, and kaolinite (trace amounts). This evolution corresponds to the mineralogic trend observed during the first stages of Cenozoic glaciation (Chamley, 1979).

During the Miocene (Hole 329, Cores 27-1; Hole 512 , Cores 5-1), smectite abundance decreases again (60 $\pm 20 \%$ ) whereas chlorite $(5 \pm 5 \%)$, illite $(15 \pm 5 \%)$, mixed-layer clays $(17.5 \pm 7.5 \%)$, and (locally) kaolinite $(5 \pm 5 \%)$ increase, following the development of glaciation. Fluctuations in the relative abundance of smectite and illite (S/I) during the Miocene are probably related to variations in climate and oceanic circulation.
Plio-Pleistocene sediments (Hole 512, Core 1) are characterized by a large increase in the abundance of smectite $(90 \%)$; illite $(5 \%)$, mixed-layer clays $(5 \%)$, chlorite, and kaolinite (trace amounts) decrease. This stratigraphic interval was poorly represented at Site 512 . If one considers the general trend of climate during late Cenozoic time, climatic determination of the clay mineral content is improbable at this site, and the occurrence of abundant smectite probably reflects detrital supply by currents.

\section{Geochemistry (Site 329, Fig. 6; Table 4)}

In upper Paleocene sediments, $\mathrm{D}^{*}(0.58)$ is very close to the value characteristic of typical shales. $\mathrm{Si}^{*}$ is low (4.11). $\mathrm{Mn}^{*}$ is always positive $(0.40)$, indicating the presence of an oxidized environment.

Oligocene and Miocene sediments show a slight increase of $\mathrm{D}^{*}(0.63)$, whereas $\mathrm{Si}^{*}(13.95)$ increases sharp- 
Table 2. Hole 330 geochemical results.

\begin{tabular}{|c|c|c|c|c|c|c|c|c|c|c|c|c|c|c|c|c|c|c|c|c|}
\hline $\begin{array}{l}\text { Core/Section } \\
\text { (level in cm) }\end{array}$ & $\begin{array}{l}\mathrm{SiO}_{2} \\
(\%)\end{array}$ & $\begin{array}{c}\mathrm{Al}_{2} \mathrm{O}_{3} \\
(\%)\end{array}$ & $\begin{array}{l}\mathrm{CaO} \\
(\%)\end{array}$ & $\begin{array}{c}\mathrm{MgO} \\
(\%)\end{array}$ & $\begin{array}{c}\mathrm{Na}_{2} \mathrm{O} \\
(\%)\end{array}$ & $\begin{array}{l}\mathrm{K}_{2} \mathrm{O} \\
(\%)\end{array}$ & $\begin{array}{l}\mathrm{TiO}_{2} \\
(\%)\end{array}$ & $\begin{array}{c}\mathrm{P}_{2} \mathrm{O}_{5} \\
(\%)\end{array}$ & $\begin{array}{c}\mathrm{Fe}_{2} \mathrm{O}_{3} \\
(\%)\end{array}$ & $\underset{(\mathrm{ppm})}{\mathrm{Mn}}$ & $\underset{(\mathrm{ppm})}{\mathrm{Zn}}$ & $\underset{(\mathrm{ppm})}{\mathrm{Li}}$ & $\underset{(\mathrm{ppm})}{\mathrm{Ni}}$ & $\underset{(\mathrm{ppm})}{\mathrm{Cr}}$ & $\begin{array}{c}\mathrm{Sr} \\
(\mathrm{ppm})\end{array}$ & $\begin{array}{c}\text { Co } \\
\text { (ppm) }\end{array}$ & $\underset{(\mathrm{ppm})}{\mathrm{Cu}}$ & $\begin{array}{c}\mathrm{Pb} \\
(\mathrm{ppm})\end{array}$ & $\underset{(\mathrm{ppm})}{\mathrm{V}}$ & $\underset{(\mathrm{ppm})}{\mathrm{Cd}}$ \\
\hline $1-1,46$ & 26.90 & 6.22 & 31.47 & 1.25 & 1.48 & 1.99 & 0.22 & n.d. & 2.97 & 1968 & 174 & 29 & 36 & 26 & 332 & 15 & 16 & 33 & 21 & 0 \\
\hline $1-2,103$ & 33.35 & 7.77 & 24.99 & 1.55 & 1.80 & 2.20 & 0.18 & n.d. & 3.22 & 1610 & 253 & 36 & 35 & 26 & 337 & 11 & 28 & 33 & 26 & 0 \\
\hline $1-3,148$ & 30.75 & 6.92 & 26.84 & 1.50 & 2.29 & 2.21 & 0.25 & n.d. & 2.64 & 1568 & 332 & 28 & 26 & 26 & 316 & 9 & 19 & 32 & 21 & 1 \\
\hline $1-5,47$ & 22.80 & 5.35 & 35.17 & 1.20 & 1.15 & 1.61 & 0.15 & n.d. & 2.19 & 2168 & 263 & 23 & 25 & 25 & 300 & 11 & 13 & 28 & 18 & 0 \\
\hline $1-6,96$ & 28.50 & 6.25 & 30.54 & 1.35 & 1.48 & 1.91 & 0.23 & n.d. & 2.86 & 2221 & 268 & 26 & 29 & 26 & 300 & 11 & 12 & 36 & 21 & 0 \\
\hline $2-2,139$ & 43.75 & 10.04 & 15.04 & 2.47 & 2.63 & 1.69 & 0.41 & n.d. & 3.50 & 2684 & 243 & 79 & 49 & 31 & 316 & 21 & 6 & 55 & 32 & 5 \\
\hline $3-2,86$ & 4.45 & 0.83 & 48.45 & 0.75 & 0.04 & 0.12 & 0.03 & n.d. & 1.43 & 9250 & 491 & 4 & 23 & 19 & 437 & 9 & 15 & 42 & 158 & 20 \\
\hline $4-2,142$ & 58.80 & 14.60 & 0.42 & 1.91 & 0.91 & 3.70 & 0.52 & n.d. & 5.33 & 379 & 268 & 56 & 23 & 97 & 105 & 4 & 14 & 27 & 132 & 1 \\
\hline $5-2,77$ & 53.50 & 14.64 & 1.82 & 1.79 & 0.91 & 2.86 & 0.52 & n.d. & 5.65 & 247 & 531 & 50 & 90 & 74 & 142 & 2 & 29 & 31 & 426 & 5 \\
\hline $5-3,117$ & 50.50 & 14.05 & 4.79 & 1.62 & 0.81 & 3.25 & 0.48 & n.d. & 5.26 & 232 & 394 & 44 & 83 & 61 & 158 & 1 & 26 & 38 & 374 & 4 \\
\hline $6-1,48$ & 54.65 & 14.64 & 2.94 & 1.81 & 0.88 & 3.61 & 0.49 & n.d. & 5.33 & 237 & 375 & 50 & 80 & 74 & 126 & 2 & 25 & 33 & 311 & 3 \\
\hline $6-2,95$ & 53.75 & 14.29 & 3.04 & 1.76 & 0.91 & 3.37 & 0.48 & n.d. & 5.43 & 221 & 578 & 47 & 105 & 75 & 158 & 5 & 37 & 35 & 458 & 5 \\
\hline $6-4,52$ & 54.25 & 14.50 & 3.64 & 1.78 & 0.94 & 2.86 & 0.48 & n.d. & 5.12 & 226 & 660 & 47 & 87 & 67 & 179 & 2 & 22 & 54 & 347 & 6 \\
\hline $6-5,102$ & 53.10 & 14.42 & 3.50 & 1.81 & 0.84 & 3.40 & 0.46 & n.d. & 5.65 & 232 & 555 & 45 & 83 & 75 & 168 & 2 & 24 & 37 & 516 & 5 \\
\hline $6-6,141$ & 52.80 & 14.32 & 3.15 & 1.80 & 1.11 & 3.31 & 0.43 & n.d. & 5.86 & 232 & 595 & 49 & 97 & 79 & 137 & 4 & 36 & 35 & 500 & 5 \\
\hline $7-2,51$ & 54.75 & 14.81 & 2.03 & 1.83 & 1.01 & 3.25 & 0.54 & n.d. & 6.58 & 258 & 572 & 53 & 93 & 83 & 132 & 4 & 27 & 34 & 389 & 3 \\
\hline $7-3,94$ & 53.60 & 14.61 & 2.52 & 1.72 & 1.11 & 3.37 & 0.56 & n.d. & 5.76 & 237 & 634 & 53 & 91 & 91 & 142 & 3 & 25 & 32 & 553 & 6 \\
\hline $7-4,145$ & 52.80 & 14.69 & 2.34 & 1.79 & 1.11 & 3.28 & 0.48 & n.d. & 6.43 & 221 & 352 & 58 & 85 & 79 & 126 & 5 & 23 & 31 & 442 & 3 \\
\hline $7-6,46$ & 47.80 & 13.11 & 6.96 & 1.61 & 0.88 & 3.10 & 0.48 & n.d. & 5.62 & 237 & 442 & 44 & 86 & 74 & 174 & 1 & 28 & 35 & 416 & 5 \\
\hline $8-1,91$ & 51.05 & 14.15 & 3.71 & 1.76 & 1.08 & 3.25 & 0.48 & n.d. & 5.86 & 221 & 498 & 52 & 80 & 75 & 158 & 5 & 31 & 34 & 421 & 4 \\
\hline $8-3,55$ & 55.60 & 14.64 & 1.96 & 1.90 & 1.08 & 3.10 & 0.35 & n.d. & 5.26 & 226 & 337 & 51 & 51 & 113 & 158 & 2 & 26 & 32 & 421 & 3 \\
\hline $8-4,104$ & 57.05 & 15.08 & 0.35 & 1.91 & 1.08 & 3.30 & 0.50 & n.d. & 5.12 & 200 & 263 & 48 & 19 & 126 & 142 & 0 & 26 & 33 & 211 & 0 \\
\hline $9-1,109$ & 56.75 & 14.86 & 0.73 & 1.91 & 1.18 & 3.19 & 0.54 & n.d. & 5.72 & 195 & 277 & 47 & 16 & 111 & 179 & 0 & 22 & 32 & 158 & 0 \\
\hline $9-2,139$ & 57.40 & 15.53 & 0.28 & 1.94 & 1.01 & 3.19 & 0.52 & n.d. & 6.19 & 232 & 302 & 54 & 16 & 102 & 153 & 2 & 21 & 36 & 158 & 0 \\
\hline $10-1,103$ & 57.35 & 15.48 & 0.35 & 2.03 & 1.42 & 2.71 & 0.52 & n.d. & 5.93 & 211 & 287 & 46 & 100 & 118 & 179 & 0 & 31 & 35 & 189 & 0 \\
\hline $10-2,147$ & 58.50 & 15.46 & 0.17 & 2.01 & 1.25 & 2.89 & 0.55 & n.d. & 6.76 & 200 & 361 & 56 & 27 & 111 & 153 & 3 & 19 & 34 & 142 & 2 \\
\hline $11-1,64$ & 53.95 & 16.89 & 1.33 & 1.29 & 1.04 & 2.80 & 0.63 & 0.56 & 6.22 & 284 & 322 & 89 & 21 & 104 & 179 & 8 & 20 & 40 & 168 & 1 \\
\hline $11-2,96$ & 56.65 & 16.73 & 0.35 & 1.26 & 0.98 & 2.98 & 0.69 & n.d. & 6.79 & 258 & 323 & 96 & 18 & 113 & 100 & 11 & 22 & 33 & 126 & 1 \\
\hline $11-4,7$ & 58.40 & 16.70 & 0.21 & 1.33 & 1.01 & 3.13 & 0.73 & n.d. & 5.76 & 247 & 357 & 91 & 16 & 108 & III & 6 & 19 & 33 & 111 & 0 \\
\hline $11-5,47$ & 59.45 & 15.41 & 0.31 & 1.21 & 0.94 & 3.07 & 0.65 & n.d. & 6.22 & 221 & 262 & 82 & 11 & 105 & 105 & 5 & 17 & 111 & 89 & 0 \\
\hline $11-6,101$ & 58.00 & 16.64 & 0.31 & 1.15 & 0.88 & 2.98 & 0.74 & n.d. & 5.72 & 237 & 376 & 94 & 15 & 113 & 95 & 5 & 20 & 39 & 105 & 0 \\
\hline $12-1,147$ & 57.85 & 16.95 & 0.35 & 1.37 & 1.15 & 3.13 & 0.71 & n.d. & 5.98 & 258 & 273 & 101 & 21 & 100 & 95 & 7 & 26 & 36 & 89 & 0 \\
\hline $12-3,52$ & 58.10 & 15.93 & 0.35 & 1.24 & 0.91 & 3.16 & 0.69 & n.d. & 6.33 & 237 & 267 & 91 & 11 & 104 & 105 & 11 & 23 & 32 & 142 & 0 \\
\hline $12-4,103$ & 57.55 & 15.46 & 0.31 & 1.16 & 0.91 & 2.74 & 0.65 & n.d. & 6.36 & 295 & 248 & 93 & 15 & 113 & 105 & 6 & 24 & 34 & 126 & 0 \\
\hline $12-6,54$ & 59.80 & 15.35 & 0.28 & 1.32 & 0.88 & 3.04 & 0.75 & n.d. & 5.83 & 289 & 207 & 87 & 15 & 104 & 116 & 10 & 23 & 33 & 121 & 0 \\
\hline $13-1,97$ & 59.15 & 14.24 & 0.56 & 1.32 & 1.01 & 2.74 & 0.69 & n.d. & 6.22 & 374 & 447 & 80 & 11 & 93 & 126 & 6 & 17 & 34 & 95 & 0 \\
\hline $13-2,146$ & 64.90 & 13.18 & 0.49 & 0.99 & 1.21 & 2.71 & 0.71 & n.d. & 4.65 & 216 & 232 & 63 & 10 & 111 & 105 & 8 & 7 & 44 & 95 & 0 \\
\hline $13-4,46$ & 62.60 & 13.93 & 0.31 & 0.99 & 0.98 & 2.92 & 0.79 & n.d. & 6.55 & 221 & 167 & 54 & 5 & 94 & 89 & 7 & 3 & 31 & 95 & 0 \\
\hline $14-1,98$ & 61.70 & 13.93 & 0.66 & 0.88 & 0.88 & 3.16 & 0.82 & n.d. & 7.83 & 626 & 192 & 52 & 0 & 103 & 95 & 9 & 0 & 32 & 121 & 0 \\
\hline $14-2,133$ & 58.35 & 15.69 & 0.63 & 0.99 & 1.04 & 3.07 & 0.85 & n.d. & 7.72 & 484 & 188 & 71 & 6 & 113 & 95 & 12 & 5 & 32 & 116 & 0 \\
\hline $14-4,52$ & 55.85 & 16.79 & 0.52 & 1.00 & 0.91 & 2.92 & 0.79 & n.d. & 8.48 & 289 & 209 & 79 & 5 & 118 & 105 & 7 & 11 & 27 & 121 & 0 \\
\hline $15-2,35$ & 75.30 & 8.08 & 0.24 & 0.36 & 0.74 & 1.84 & 0.35 & n.d. & 1.93 & 74 & 105 & 19 & 0 & 39 & 121 & 0 & 0 & 46 & 5 & 0 \\
\hline $15, \mathrm{CC}$ & 70.95 & 15.21 & 0 & 0.41 & 0.30 & 2.86 & 0.36 & n.d. & 2.07 & 32 & 205 & 52 & 0 & 55 & 47 & 0 & 9 & 41 & 21 & 0 \\
\hline $16-1,31$ & 79.70 & 7.91 & 1.01 & 0.61 & 0.67 & 2.20 & 0.63 & n.d. & 3.57 & 111 & 191 & 30 & 0 & 79 & 79 & 0 & 232 & 101 & 42 & 0 \\
\hline
\end{tabular}

Note: n.d. $=$ no data .

ly. $\mathrm{Mn}^{*}$ values fluctuate above and below zero. The increase of $\mathrm{Si}^{*}$ may be related to a detrital supply of silica or to an increase in siliceous planktonic productivity. This increase occurred during the opening of the Drake Passage, which resulted in both increased detrital supply by Pacific currents and greater siliceous productivity.

\section{GEORGIA BASIN}

Site 328

\section{Lithology (Fig. 8)}

The base of the sedimentary section, from upper Turonian to Maestrichtian, consists of variegated claystones overlain by zeolitic clays and claystones from the Maestrichtian to the Eocene/Oligocene boundary. Oligocene and Miocene sediments consist of siliceous oozes, clayey oozes, and zeolitic clays. Pliocene and Pleistocene sediments are represented by diatom oozes with ice-rafted detritus.

\section{Clay Mineralogy}

In upper Turonian to upper Eocene sediments (Hole 328B, Cores 8-7; Hole 328, Cores 12-5), smectite represents almost $100 \%$ of the clay fraction, accompanied by trace amounts of illite, mixed-layer clays, and kaolinite. The persistently high smectite content through a long stratigraphic interval points to the persistence of both a hot climate with major seasonal changes in humidity and a low relief on the adjacent continents.

In upper Eocene, Oligocene, and Miocene sediments (Hole 328B, Cores 6-1), only a minor increase occurs in the abundances of illite $(5 \pm 5 \%)$, mixed-layer clays $(2.5 \pm 2.5 \%)$, and kaolinite $(5 \pm 5 \%)$; smectite abundance diminishes $(90 \pm 5 \%)$. In the Georgia Basin, the Cenozoic increase in primary minerals and mixed-layer clays related to the development of glaciation is less significant than it is on the Falkland Plateau (Sites 329 and 512 , principally).

In general, the abundance of smectite in the Georgia Basin is very significant when compared to the amounts found on the Falkland Plateau or the Mid-Atlantic Ridge, especially in Cenozoic sediments.

\section{MID-ATLANTIC RIDGE}

Sites 513, 514

Lithology (Figs. 9-10)

The base of the sedimentary section (Site 513) consists of a lower Oligocene chert fragment overlying basaltic basement. A nannofossil ooze is present from the lower to the upper Oligocene, and the uppermost Oligocene and lower Miocene are represented by interlayered diatomaceous nannofossil oozes and nannofossil oozes. From the middle Miocene to the Pleistocene, the sedi- 
Table 3. Hole 327A geochemical results.

\begin{tabular}{|c|c|c|c|c|c|c|c|c|c|c|c|c|c|c|c|c|c|c|c|c|}
\hline $\begin{array}{l}\text { Core/Section } \\
\text { (level in } \mathrm{cm} \text { ) }\end{array}$ & $\begin{array}{l}\mathrm{SiO}_{2} \\
(\%)\end{array}$ & $\begin{array}{l}\mathrm{Al}_{2} \mathrm{O}_{3} \\
(\%)\end{array}$ & $\begin{array}{l}\mathrm{CaO} \\
(\%)\end{array}$ & $\begin{array}{l}\mathrm{MgO} \\
(\%)\end{array}$ & $\underset{(\%)}{\mathrm{Na}_{2} \mathrm{O}}$ & $\begin{array}{l}\mathrm{K}_{2} \mathrm{O} \\
(\%)\end{array}$ & $\begin{array}{l}\mathrm{TiO}_{2} \\
(\%)\end{array}$ & $\begin{array}{l}\mathrm{P}_{2} \mathrm{O}_{5} \\
(\%)\end{array}$ & $\begin{array}{l}\mathrm{Fe}_{2} \mathrm{O}_{3} \\
(\%)\end{array}$ & $\begin{array}{c}\mathrm{Mn} \\
(\mathrm{ppm})\end{array}$ & $\underset{(\mathrm{ppm})}{\mathrm{Zn}}$ & $\underset{(\mathrm{ppm})}{\mathrm{Li}}$ & $\underset{(\mathrm{ppm})}{\mathrm{Ni}}$ & $\underset{(\mathrm{ppm})}{\mathrm{Cr}}$ & $\underset{(\mathrm{ppm})}{\mathrm{Sr}}$ & $\begin{array}{c}\text { Co } \\
(\mathrm{ppm})\end{array}$ & $\underset{(\mathrm{ppm})}{\mathrm{Cu}}$ & $\begin{array}{c}\mathrm{Pb} \\
(\mathrm{ppm})\end{array}$ & $\underset{(\mathrm{ppm})}{\mathrm{V}}$ & $\underset{(\mathrm{ppm})}{\mathrm{Cd}}$ \\
\hline $1-2,51$ & 72.05 & 6.73 & 1.41 & 1.41 & 1.75 & 2.11 & 0.45 & n.d. & 7.22 & 568 & 183 & 18 & 80 & 72 & 179 & 32 & 29 & 177 & 53 & n.d. \\
\hline $2-1,88$ & 51.55 & 10.16 & 2.20 & 2.75 & 3.34 & 3.01 & 0.33 & 0.60 & 11.47 & 453 & 240 & 48 & 142 & 51 & 226 & 30 & 64 & 53 & 100 & n.d. \\
\hline $2-2,148$ & 51.30 & 10.45 & 1.76 & 2.74 & 2.80 & 2.95 & 0.23 & 0.58 & 11.01 & 1432 & 446 & so & 142 & 49 & 221 & 27 & 84 & 44 & 89 & n.d. \\
\hline $2-4,51$ & 52.80 & 11.63 & 1.48 & 3.32 & 3.12 & 2.89 & 0.49 & 0.71 & 10.87 & 974 & 356 & 61 & 84 & 44 & 226 & 14 & 82 & 36 & 63 & n.d. \\
\hline $2-5,97$ & 52.40 & 11.22 & 1.53 & 2.57 & 3.25 & 3.18 & 0.21 & 1.09 & 10.12 & 144 & 192 & 50 & 86 & 44 & 242 & 16 & 75 & 51 & 84 & n.d. \\
\hline $5-1,20$ & 45.05 & 8.56 & 12.00 & 2.12 & 3.84 & 1.60 & 0.33 & n.d. & 4.22 & 158 & 114 & 44 & 4 & 28 & 411 & 1 & 6 & 34 & 16 & n.d. \\
\hline $5-2,98$ & 45.45 & 10.04 & 8.50 & 2.63 & 4.72 & 1.93 & 0.38 & n.d. & 4.25 & 458 & $\begin{array}{l}114 \\
215\end{array}$ & 88 & 45 & 37 & 332 & 11 & 13 & 49 & 53 & n.d. \\
\hline $5-3,148$ & 47.20 & 8.74 & 10.00 & 2.25 & 4.08 & $\begin{array}{l}1.69 \\
\text {. }\end{array}$ & 0.31 & n.d. & 3.86 & 195 & 145 & 88 & 25 & 38 & 363 & 9 & 16 & 41 & 42 & n.d. \\
\hline $5-5,51$ & $\begin{array}{r}49.05 \\
\end{array}$ & $\begin{array}{l}8.14 \\
9.33\end{array}$ & 8.22 & 2.07 & $\begin{array}{l}4.06 \\
4.26\end{array}$ & $\begin{array}{l}1.70 \\
\end{array}$ & 0.40 & n.d. & $\begin{array}{l}3.00 \\
3.43\end{array}$ & 153 & 126 & $\begin{array}{l}80 \\
108\end{array}$ & 37 & 42 & 326 & 13 & 27 & 46 & 79 & n.d. \\
\hline $5-6,97$ & 46.60 & 10.22 & 9.51 & 2.16 & 3.57 & 1.87 & 0.47 & n.d. & 4.22 & 184 & 116 & 115 & 34 & 44 & 347 & 10 & 26 & 53 & 63 & n.d. \\
\hline $6-3,51$ & 53.05 & 10.81 & 5.00 & 2.35 & 4.11 & 1.90 & 0.33 & n.d. & 5.33 & 274 & 162 & 97 & 33 & 37 & 263 & 11 & 14 & 33 & 63 & n.d. \\
\hline $6-4,97$ & 54.95 & 10.45 & 4.07 & 2.17 & 3.49 & 1.79 & 0.56 & n.d. & 5.15 & 221 & 217 & 99 & 25 & 42 & 242 & 9 & 25 & 35 & 68 & n.d. \\
\hline $6-5,148$ & 50.90 & 10.75 & 6.65 & 2.51 & 4.00 & 1.97 & 0.45 & n.d. & 4.43 & 158 & 221 & 109 & 38 & 44 & 279 & 9 & 21 & 37 & 37 & n.d. \\
\hline $7-2,51$ & 57.60 & 9.09 & 1.28 & 2.29 & 4.84 & 1.85 & 0.53 & n.d. & 4.65 & 211 & 114 & 99 & 28 & 43 & 153 & 7 & 21 & 37 & 16 & n.d. \\
\hline $8-2,148$ & 53.50 & 13.11 & 0.87 & 2.35 & 3.54 & 2.62 & 0.73 & n.d. & 5.97 & 137 & 171 & 88 & 19 & 70 & 168 & 9 & 34 & 34 & 74 & n.d. \\
\hline $8-4,49$ & 53.35 & 11.69 & 0.65 & 2.90 & 3.62 & 2.67 & 0.65 & n.d. & 6.04 & 126 & 149 & 58 & 23 & 71 & 526 & 8 & 28 & 33 & 63 & n.d. \\
\hline $8-5,97$ & 59.90 & 8.44 & 0.76 & 2.16 & 2.88 & 2.21 & 0.50 & n.d. & 5.61 & 100 & 149 & 45 & 18 & 68 & 153 & 4 & 13 & 25 & 42 & n.d. \\
\hline $9-1,148$ & 38.35 & 8.38 & 0.63 & 2.79 & 3.83 & 2.29 & 0.55 & n.d. & $\begin{array}{r}1.01 \\
14.73\end{array}$ & 132 & 189 & 76 & 79 & 42 & 179 & 7 & 13 & 32 & 32 & n.d. \\
\hline $9-3,49$ & 50.35 & $\begin{array}{r}8.36 \\
11.24\end{array}$ & $\begin{array}{l}0.03 \\
0.92\end{array}$ & 3.09 & $\begin{array}{l}3.03 \\
4.08\end{array}$ & $\begin{array}{l}2.29 \\
1.87\end{array}$ & 0.58 & $\begin{array}{l}\text { n.d. } \\
\text { n. }\end{array}$ & $\begin{array}{r}14.53 \\
6.58\end{array}$ & $\begin{array}{l}158 \\
158\end{array}$ & 226 & 92 & 42 & 53 & 174 & 7 & 32 & 29 & 79 & 0 \\
\hline $9-4,103$ & 61.75 & 8.35 & 0.48 & 1.87 & 2.29 & 1.51 & 0.43 & n.d. & 4.93 & 89 & 242 & 37 & 43 & 72 & 95 & 4 & 56 & 19 & 53 & 0 \\
\hline $9-5,148$ & 53.70 & 10.68 & 0.73 & $\begin{array}{l}2.79 \\
2.79\end{array}$ & 3.74 & 2.80 & $\begin{array}{l}0.73 \\
0.72\end{array}$ & n.d. & $\begin{array}{l}4.95 \\
7.51\end{array}$ & 116 & 374 & 62 & 92 & 128 & $\begin{array}{l}95 \\
168\end{array}$ & 23 & $\begin{array}{l}30 \\
99\end{array}$ & 32 & 132 & 0 \\
\hline $10-3,48$ & 26.30 & $\begin{array}{r}4.06 \\
4.78\end{array}$ & 31.38 & 1.25 & $\begin{array}{l}3.49 \\
1.49\end{array}$ & $\begin{array}{l}2.80 \\
0.79\end{array}$ & 0.22 & n.d. & 2.62 & 663 & 205 & 24 & 43 & 31 & 916 & 17 & 23 & 49 & 132 & 7 \\
\hline $11-1,58$ & 27.80 & 4.99 & 28.93 & 1.43 & 2.02 & 0.76 & 0.23 & n.d. & 3.19 & 574 & 384 & 33 & 37 & 28 & 874 & 14 & 22 & 40 & 132 & 7 \\
\hline $11-2,122$ & 65.30 & 2.72 & 12.24 & 0.63 & 1.25 & 0.60 & 0.10 & n.d. & 1.36 & 279 & 216 & 14 & 21 & 22 & 363 & 8 & 2 & 32 & 0 & 3 \\
\hline $12-2,48$ & 27.00 & 6.48 & 26.58 & 2.01 & 2.60 & 0.95 & 0.22 & n.d. & 3.86 & 958 & 463 & 39 & 33 & 27 & 847 & 15 & 23 & 62 & 132 & 4 \\
\hline $12-3,97$ & 29.20 & 7.05 & 23.89 & 2.24 & 3.88 & 0.76 & 0.35 & n.d. & 3.05 & 721 & 284 & 84 & 41 & 23 & 816 & 15 & 26 & 41 & 158 & 5 \\
\hline $12-4,115$ & 16.80 & 3.59 & 37.50 & 1.23 & 1.69 & 0.45 & 0.13 & n.d. & 2.22 & 2010 & 247 & 18 & 35 & 22 & 1079 & 18 & 20 & 53 & 53 & 9 \\
\hline $14-1,110$ & 43.60 & 10.92 & 13.68 & 2.40 & 2.09 & 1.69 & 0.47 & n.d. & 4.55 & 3063 & 263 & 37 & 83 & 26 & 263 & 22 & 29 & 55 & 158 & 3 \\
\hline $14-2,147$ & 55.25 & 14.15 & 1.51 & 3.04 & 2.87 & 1.93 & 0.60 & 0.34 & 8.29 & 1047 & 321 & 54 & 84 & 27 & 174 & 18 & 43 & 40 & 211 & 0 \\
\hline $14-4,48 \mathrm{a}$ & 56.25 & 15.10 & 1.26 & 2.86 & 2.97 & $\begin{array}{l}1.98 \\
1.98\end{array}$ & 0.55 & 0.4 & $\begin{array}{l}0.12 \\
6.12\end{array}$ & 1058 & 358 & 62 & 45 & 26 & 174 & 6 & 25 & 40 & 263 & 0 \\
\hline $14-4,48 b$ & $\begin{array}{l}55.75 \\
55\end{array}$ & $\begin{array}{l}14.10 \\
14.38\end{array}$ & $\begin{array}{l}1.15 \\
1.15\end{array}$ & $\begin{array}{l}2.80 \\
2.81\end{array}$ & $\begin{array}{l}2.91 \\
2.97\end{array}$ & $\begin{array}{l}1.98 \\
2.40\end{array}$ & 0.58 & 0 & $\begin{array}{l}0.12 \\
7.58\end{array}$ & $\begin{array}{r}1038 \\
895\end{array}$ & 253 & $\begin{array}{l}02 \\
57\end{array}$ & $\begin{array}{l}43 \\
58\end{array}$ & $\begin{array}{l}20 \\
37\end{array}$ & $\begin{array}{l}184 \\
184\end{array}$ & $\begin{array}{l}0 \\
17\end{array}$ & 43 & 38 & 211 & 0 \\
\hline $14-5,96$ & 34.15 & 8.03 & 21.76 & 1.81 & 1.63 & 1.72 & 0.32 & n.d. & 4.50 & $\begin{array}{r}1410 \\
1410\end{array}$ & 258 & 46 & 42 & 23 & $\begin{array}{l}184 \\
189\end{array}$ & 17 & 24 & 55 & 79 & 3 \\
\hline $14-6,148$ & 15.80 & 3.97 & 37.78 & 1.24 & 1.49 & 0.57 & 0.15 & n.d. & 1.22 & 863 & 253 & 25 & 32 & 16 & 216 & 19 & 15 & 53 & 53 & 6 \\
\hline $15-2,113$ & 35.20 & 7.48 & 21.44 & 1.68 & 2.13 & 1.47 & 0.33 & n.d. & 3.15 & 1832 & 589 & 34 & 41 & 20 & 458 & 25 & 19 & 47 & 53 & 3 \\
\hline $16-1,143$ & 35.00 & 7.01 & 22.70 & 1.75 & 2.09 & 0.91 & 0.52 & n.d. & 2.79 & 2874 & 447 & 33 & 52 & 25 & 432 & 35 & 54 & 48 & 53 & 1 \\
\hline $16-3,52$ & 33.90 & 6.59 & 24.59 & 1.62 & 1.62 & 0.85 & 0.27 & n.d. & 2.54 & 2605 & 247 & 26 & 43 & 21 & 437 & 32 & 19 & 42 & 79 & 4 \\
\hline $16-4,94$ & 31.25 & 6.69 & 24.49 & 1.62 & 1.72 & 1.03 & 0.33 & n.d. & 4.07 & 2063 & 432 & 15 & 34 & 22 & 489 & 21 & 16 & 40 & 53 & 4 \\
\hline $16-6,51$ & 34.05 & 7.29 & 22.77 & 1.55 & 2.02 & 1.15 & 0.27 & n.d. & 3.57 & 2037 & 263 & 17 & 38 & 27 & 505 & 31 & 24 & 35 & 53 & 2 \\
\hline $18-1,51$ & 26.80 & 5.27 & 30.08 & 1.18 & 1.49 & 0.88 & 0.18 & n.d. & 2.04 & 2368 & 132 & 16 & 33 & 23 & 542 & 19 & 12 & 46 & 105 & 5 \\
\hline $18-2,88$ & 31.15 & 6.67 & 25.64 & 2.06 & 1.75 & 0.57 & 0 . & $\mathrm{n}$ & 2. & 274 & 168 & 43 & 72 & 21 & 45 & 47 & 21 & 45 & 26 & 4 \\
\hline $18-3,147$ & 39.35 & 6.25 & 21.83 & 1.11 & 2.46 & 1.1 & 0.2 & $\mathrm{n}$ & 1.47 & 184 & 216 & 15 & 30 & 25 & 52 & 14 & 23 & 129 & 79 & 0 \\
\hline $18-5,50$ & 31.05 & 5.61 & 26.55 & 1.23 & 1.52 & 1.00 & 0.22 & $\mathrm{n}$. & 2.36 & 2489 & 237 & 17 & 35 & 23 & 505 & 19 & 15 & 39 & 132 & 5 \\
\hline $18-6,110$ & 27.75 & 5.78 & 28.93 & 1.37 & 1.36 & 0.79 & 0.25 & & 2.14 & 2363 & 163 & 21 & 41 & 24 & 505 & 21 & 27 & 43 & 79 & 6 \\
\hline $19-1,49$ & 36.00 & 7.03 & 22.00 & 1.20 & $\begin{array}{l}1.30 \\
1.69\end{array}$ & 1.36 & 0.30 & n.d. & 2.64 & 2116 & $\begin{array}{l}143 \\
147\end{array}$ & 20 & 34 & 25 & 453 & 1 & 19 & 42 & 79 & 5 \\
\hline $19-2,88$ & 39.75 & 8.69 & 17.70 & 2.08 & 2.02 & 1.12 & 0.27 & n.d. & 3.15 & 223 & 221 & 48 & 73 & 23 & 411 & 45 & 22 & 32 & 26 & 4 \\
\hline $20-1,102$ & 39.55 & 8.62 & 18.57 & 1.62 & 1.79 & 1.36 & 0.33 & n. & 3.72 & 2184 & 147 & 28 & 45 & 22 & 453 & 21 & 17 & 40 & 0 & 3 \\
\hline $20-2,144$ & 39. & 8.48 & 17.98 & 1.77 & 1. & 1.27 & 0.35 & $\mathrm{n}$. & 3 . & 5050 & 258 & 33 & 49 & 27 & 368 & 32 & 37 & 26 & 26 & 3 \\
\hline $21-1,139$ & 25. & 5.37 & 30.75 & 1.38 & $i$. & 0.76 & 0.25 & n. & 1.19 & 4900 & 132 & 16 & 43 & 29 & 521 & 20 & 41 & 46 & 105 & 6 \\
\hline $21-3,47$ & 33. & 6.44 & 25.08 & 1.43 & 1.37 & 0.98 & 0.27 & n.c & 2.54 & 3050 & 126 & 25 & 44 & 24 & 405 & 19 & 13 & 45 & 79 & 5 \\
\hline $21-4,100$ & 37.35 & 6.73 & 21.79 & 1.50 & 1.72 & 0.99 & 0.27 & n.c & 2.97 & 2900 & 132 & 26 & 32 & 27 & 342 & 14 & 14 & 44 & 79 & 4 \\
\hline $22-2,144$ & 69.40 & 9.37 & 1.22 & 1.51 & 1.89 & 1.45 & 0.42 & $\mathrm{n}$. & 2.8 & 284 & 489 & 40 & 233 & 35 & 284 & 23 & 95 & 34 & 158 & 0 \\
\hline $22, \mathrm{CC}$ & 58.35 & 8.26 & 7.24 & 1.32 & 1.6 & 1.36 & 0.47 & $\mathrm{n}$ & 2. & $\begin{array}{r}284 \\
4800\end{array}$ & $\begin{array}{l}409 \\
679\end{array}$ & 38 & 324 & 35 & 347 & 43 & 64 & 41 & 184 & 0 \\
\hline $23-1,85$ & 67.00 & 9.69 & 3.53 & 1.51 & $\begin{array}{l}1.0 \\
1.3\end{array}$ & $\begin{array}{l}1.720 \\
0\end{array}$ & 0.43 & n.c & 3. & 626 & 368 & 44 & 292 & 41 & 137 & 37 & 56 & 44 & 158 & 0 \\
\hline $23-2,142$ & 62 . & $\begin{array}{l}9.0 \\
8.8\end{array}$ & $\begin{array}{l}3.53 \\
1.78\end{array}$ & 2.18 & 1.2 & 1.72 & 0. & $\begin{array}{l}\mathrm{n} \\
\mathrm{n}\end{array}$ & 8. & $\begin{array}{r}0260 \\
3000\end{array}$ & $\begin{array}{l}30 \\
37\end{array}$ & 36 & $\begin{array}{l}292 \\
221\end{array}$ & $\begin{array}{l}41 \\
34\end{array}$ & 158 & 42 & $\begin{array}{l}30 \\
89\end{array}$ & 37 & 79 & 2 \\
\hline $24-1,59$ & 60 & 6.3 & 7.98 & 1. & 1. & 1. & 0. & & & 41 & 10 & 38 & 89 & 10 & it & 5 & 122 & 32 & 353 & 33 \\
\hline $24-2,103$ & 62.55 & 7.80 & 5.11 & 1. & 1. & 1. & 0 . & 0. & & 2 & 92 & 39 & 61 & 89 & 1 & 2 & 115 & 2 & 358 & 35 \\
\hline $25-1,1$ & & 3. & 33. & 0. & 0. & 0 . & 0. & 0. & 2. & 3 & 57 & 15 & 95 & 58 & 2 & 14 & 69 & 55 & 421 & 21 \\
\hline 25 & & 1.96 & 41. & 0. & 0. & 0.51 & 0. & $\mathrm{n}$. & 1.76 & 284 & 147 & 7 & 105 & 23 & 25 & 19 & 16 & 42 & 126 & 5 \\
\hline & 23. & 4.14 & 28.89 & 0.70 & 0.74 & 1.08 & 0.22 & n.c & 3.69 & 263 & 216 & 12 & 129 & 32 & 274 & 23 & 34 & 45 & 147 & $s$ \\
\hline $26-1,48$ & 47.90 & 12.60 & 7.70 & 1.29 & 1.15 & 2.77 & 0.50 & n.d. & 4.76 & 258 & 221 & 40 & 189 & 62 & 195 & 9 & 68 & 37 & 111 & 3 \\
\hline $26-2,110$ & 58.10 & 15.00 & 0.70 & 1.82 & 1.29 & 3.25 & 0.67 & n.d & 5.90 & 232 & 279 & 49 & 58 & 95 & 105 & 19 & 39 & 29 & 84 & i \\
\hline $27-1,103$ & 23.50 & 5.31 & 33.93 & 1.05 & 0.55 & 1.20 & 0.25 & 0.46 & 2.26 & 1774 & 195 & 14 & 68 & 44 & 337 & 14 & 21 & 45 & 47 & 3 \\
\hline $27-2,132$ & 58.15 & 13.58 & 0.77 & 1.93 & 1.35 & 3.19 & 0.58 & n.d. & 4.86 & 179 & 300 & 41 & 58 & 101 & 137 & 11 & 68 & 39 & 79 & 1 \\
\hline
\end{tabular}

Note: n.d. $=$ no data

Table 4. Hole 329 geochemical results.

\begin{tabular}{|c|c|c|c|c|c|c|c|c|c|c|c|c|c|c|c|c|c|c|c|c|}
\hline $\begin{array}{l}\text { Core/Section } \\
\text { (level in } \mathrm{cm} \text { ) }\end{array}$ & $\begin{array}{l}\mathrm{SiO}_{2} \\
(\%)\end{array}$ & $\underset{(\%)}{\mathrm{Al}_{2} \mathrm{O}_{3}}$ & $\begin{array}{l}\mathrm{CaO} \\
(\%)\end{array}$ & $\begin{array}{l}\mathrm{MgO} \\
(\%)\end{array}$ & $\underset{(\%)}{\mathrm{Na}_{2} \mathrm{O}}$ & $\begin{array}{l}\mathrm{K}_{2} \mathrm{O} \\
(\%)\end{array}$ & $\begin{array}{l}\mathrm{TiO}_{2} \\
(\%)\end{array}$ & $\begin{array}{c}\mathrm{P}_{2} \mathrm{O}_{5} \\
(\%)\end{array}$ & $\begin{array}{c}\mathrm{Fe}_{2} \mathrm{O}_{3} \\
(\%)\end{array}$ & $\begin{array}{c}\mathrm{Mn} \\
(\mathrm{ppm})\end{array}$ & $\underset{(\mathrm{ppm})}{\mathrm{Zn}}$ & $\underset{(\mathrm{ppm})}{\mathrm{Li}}$ & $\begin{array}{c}\mathrm{Ni} \\
(\mathrm{ppm})\end{array}$ & $\underset{(\mathrm{ppm})}{\mathrm{Cr}}$ & $\begin{array}{c}\mathrm{Sr} \\
(\mathrm{ppm})\end{array}$ & $\begin{array}{c}\text { Co } \\
\text { (ppm) }\end{array}$ & $\underset{(\mathrm{ppm})}{\mathrm{Cu}}$ & $\begin{array}{c}\mathrm{Pb} \\
(\mathrm{ppm})\end{array}$ & $\begin{array}{c}\mathrm{V} \\
(\mathrm{ppm})\end{array}$ & $\begin{array}{c}\mathrm{Cd} \\
(\mathrm{ppm})\end{array}$ \\
\hline $20-4,148$ & 37.40 & 2.34 & 26.34 & 0.60 & 2.02 & 0.54 & 0.08 & n.d. & 0.97 & 137 & 253 & 8 & 5 & 21 & 958 & 0 & 15 & 11 & 21 & 1 \\
\hline $20-6,46$ & 45.85 & 2.27 & 21.13 & 0.70 & 2.90 & 0.48 & 0.10 & n.d. & 1.07 & 89 & 289 & 10 & 4 & 21 & 811 & 0 & II & 13 & 16 & 0 \\
\hline $21-3,50$ & 59.65 & 4.02 & 9.76 & 0.90 & 2.94 & 0.81 & 0.23 & n.d. & 1.57 & 111 & 189 & 18 & 0 & 24 & 495 & 0 & 21 & 11 & 32 & 0 \\
\hline $22-3,55$ & 67.40 & 5.01 & 4.27 & 1.05 & 2.93 & 1.05 & 0.25 & n.d. & 2.07 & 126 & 195 & 25 & 6 & 24 & 300 & 0 & 23 & 7 & 39 & 0 \\
\hline $23-2,102$ & 24.10 & 3.08 & 31.83 & 1.10 & 1.49 & 0.63 & 0.15 & n.d. & 1.33 & 137 & 153 & 13 & 10 & 25 & 1410 & 2 & 18 & 20 & 32 & 1 \\
\hline $25, \mathrm{CC}$ & 43.40 & 3.89 & 20.57 & 0.85 & 1.92 & 0.78 & 0.18 & n.d. & 1.76 & 126 & 295 & 18 & 9 & 26 & 947 & 0 & 22 & 11 & 37 & 0 \\
\hline $26-3,104$ & 69.95 & 3.67 & 1.47 & 0.90 & 3.88 & 0.78 & 0.15 & n.d. & 1.62 & 84 & 147 & 19 & 0 & 19 & 211 & 0 & 15 & 0 & 29 & 0 \\
\hline $31, \mathrm{CC}$ & 28.95 & 3.19 & 30.05 & 0.75 & 0.66 & 0.73 & 0.13 & n.d. & 1.64 & 342 & 163 & 16 & 12 & 25 & 895 & 2 & 0 & 11 & 18 & 0 \\
\hline $33-1,97$ & 8.70 & 2.32 & 40.61 & 0.75 & 0.65 & 0.43 & 0.08 & n.d. & 1.07 & 316 & 174 & 12 & 15 & 19 & 910 & 5 & 9 & 17 & 8 & 2 \\
\hline $33-2,148$ & 11.90 & 2.32 & 36.03 & 0.75 & 0.68 & 0.43 & 0.05 & n.d. & 1.12 & 374 & 211 & 10 & 12 & 22 & 979 & 4 & 3 & 22 & 11 & 2 \\
\hline $33-4,98$ & 12.35 & 3.59 & 33.93 & 1.05 & 0.92 & 0.41 & 0.17 & n.d. & 1.40 & 295 & 305 & 15 & 14 & 23 & 963 & 6 & 7 & 13 & 45 & 2 \\
\hline
\end{tabular}

Note: n.d. $=$ no data 
ment consists of muddy diatomaceous oozes and diatomaceous muds.

\section{Clay Mineralogy (Figs. 9-10)}

In lower Oligocene to upper Miocene sediments (Hole $513 \mathrm{~A}$, Cores $33-11)$, smectite is the major mineral ( 80 $\pm 10 \%)$, accompanied by illite $(10 \pm 5 \%)$, mixed-layer clays $(7.5 \pm 2.5 \%)$, attapulgite $(2.5 \pm 2.5 \%)$, chlorite, and kaolinite (trace amounts). Locally, some trace amounts of sepiolite occur. The presence of primary minerals and mixed-layer clays in appreciable amounts seems to result from a climatic change-the initiation of Cenozoic cooling during late Eocene time (Shackleton and Kennett, 1975; Chamley, 1979).

During the late Miocene and a part of the Pliocene (Hole 513, Core 9, and Hole 513A, Cores 10-1; Hole 514 , Cores 35-13), smectite remains the major mineral $(55 \pm 25 \%)$. Illite $(17.5 \pm 7.5 \%)$, chlorite $(10 \pm 10 \%)$, mixed-layer clays $(10 \pm 5 \%)$, kaolinite $(5 \pm 5 \%)$, attapulgite $(5 \pm 5 \%)$, and sepiolite (trace amounts) are also present. These data point to a deterioration in the climatic conditions during and after the late Miocene.

In upper Pliocene to Pleistocene sediments at Site 513 (Hole 513, Cores 6-1), the abundance of chlorite (12.5 $\pm 7.5 \%)$, illite $(17.5 \pm 7.5 \%)$, and mixed-layer clays $(12.5 \pm 2.5 \%)$ continues to increase; smectite abundance decreases $(50 \pm 20 \%)$. Kaolinite $(7.5 \pm 7.5 \%)$ and attapulgite (trace amounts) are also present. This trend of the clay mineralogy follows the development of Cenozoic glaciation. In Hole 514 (Cores 13-1) smectite abundance increases slightly $(55 \pm 25 \%)$. Chlorite $(12.5$ $\pm 7.5 \%)$, illite $(17.5 \pm 7.5 \%)$, mixed-layer clays $(7.5$ $\pm 2.5 \%)$, kaolinite $(5 \pm 5 \%)$, and attapulgite $(2.5 \pm$ $2.5 \%$ ) are also present. The conflicting evolution of the clay mineral associations at Sites 513 and 514 is also present in the S/I. Whereas the climate is dominated by the development of the late Cenozoic glaciation, the mineralogical changes observed certainly indicate a significant influence of oceanic currents (Robert, 1980).

\section{Geochemistry (Site 513, Fig. 9; Table 5)}

During Oligocene time, $\mathrm{D}^{*}$ is high $(0.60) . \mathrm{Si}^{*}$ is very high in the lower Oligocene (16.54) and progressively decreases to 6.73 in the upper Oligocene. These values suggest a strong detrital input. High positive $\mathrm{Mn}^{*}$ values $(0.82)$ in the lower Oligocene decrease slightly toward the upper Oligocene (0.52); these high values are almost certainly related to volcanic events along the rift, which were very influential during the early Oligocene but which later decreased when Site 513 edged away from the rift area. $\mathrm{Mg}^{*}$, very high during early Oligocene time $(0.48)$, decreased afterward $(0.26)$; this trend can be explained by the chemical composition of calcite minerals. In the lower Oligocene, $\mathrm{Sr}^{*}(2.85)$ is typical of marine carbonates. Values increase in the upper Oligocene, suggesting a slow dissolution of the carbonate fraction.

In Miocene sediments, $D^{*}$ increases slightly $(0.62)$, suggesting that terrigenous components had greater influence. In the lower Miocene, $\mathrm{Mn}^{*}$ is very close to its Oligocene values, but a major break occurs between Cores 14 and $13(0.12)$. Simultaneously, $\mathrm{Mg}^{*}$ decreases upward through the Miocene sediments. $\mathrm{Sr}^{*}$ is relatively low in the lowermost Miocene and increases considerably in Core 12 and above. It is suggested that Site 513 passed through the CCD by seafloor subsidence or by elevation of the CCD itself. These modifications in the chemical characteristics of the sediments occurred at the time that the Drake Passage opened, inducing an increased supply of detrital material from southern regions that diluted the volcanic components originating from the rift.

In Plio-Pleistocene sediments, $\mathrm{D}^{*}$ increases slightly again (0.64). High values are clearly evident at the top of the Pleistocene, in agreement with the data of Lisitzin (1962) and Angino (1964) concerning pelagic circumAntarctic sediments. $\mathrm{Mn}^{*}$ becomes slightly negative $(-0.07)$. The lower sedimentation rates would normally favor an increased contribution from volcanogenic components, but the increasing distance of Site 513 from the rift meant that such material had little apparent influence. The increase in $\mathrm{Sr}^{*}(10.77-14.36)$ points to a strong dissolution of carbonates-Site 513 is located below the CCD.

\section{PALEOGEOGRAPHIC AND PALEOCEANO- GRAPHIC EVOLUTION OF THE SOUTHERN OCEAN AND FALKLAND PLATEAU}

The variations in clay mineral assemblages and geochemical indices permit us to reconstruct several stages in the evolution of the South Atlantic.

In the Early Jurassic reconstruction of Gondwanaland, the present-day Falkland Plateau was located along the southeastern coast of South Africa, near the Mozambique Plateau (Craddock, 1970; Thompson, 1976; De Wit, 1977). The first marine incursion reached the Maurice Ewing Bank during Middle to Late Jurassic time (Barker, Dalziel, et al., 1977).

Sediments of this age are characterized by a preponderance of detrital components; the continental environment exercised major influence. The organic matter, continental in origin (Herbin and Deroo, 1979), favors the dissolution of the carbonates, but the strontium released by dissolution is trapped in the sediment.

The clay fraction from Middle to Upper Jurassic sediments contains major amounts of chlorite, illite, mixedlayer clays, and kaolinite (Fig. 11). This mineral assemblage indicates intense erosion of altered rocks and of moderately to deeply weathered soils from areas of high relief. It suggests the presence of a youthful topography, rejuvenated by tectonism. Similar mineral associations occur in lower Aptian sediments in the Cape Basin and in upper Aptian-lower Albian sediments of the Angola Basin (Robert et al., 1979), where they indicate marginal instability and tectonic activity, related to the initial opening of the oceanic basins. In the Falkland Plateau area, the Middle to Late Jurassic mineral assemblage marks the first stage of the opening of the Southern Ocean.

At the end of the Middle Jurassic, a reducing, shallow, marine environment appears, which partially washes the strontium released during the dissolution of the carbonates by organic acids. 


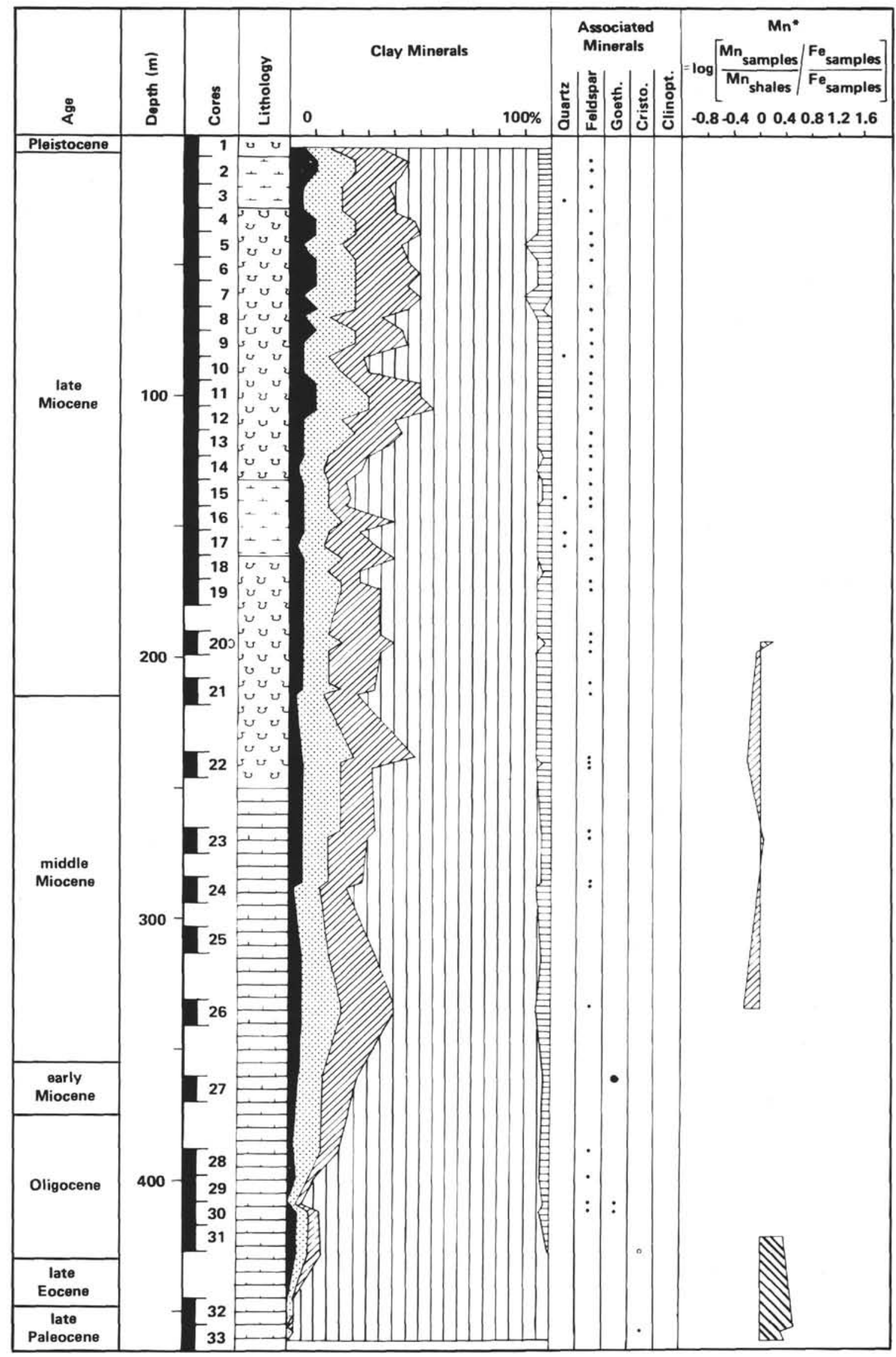

Figure 6. Hole 329 results. 
PALEOENVIRONMENTAL SIGNIFICANCE OF CLAY MINERAL DATA

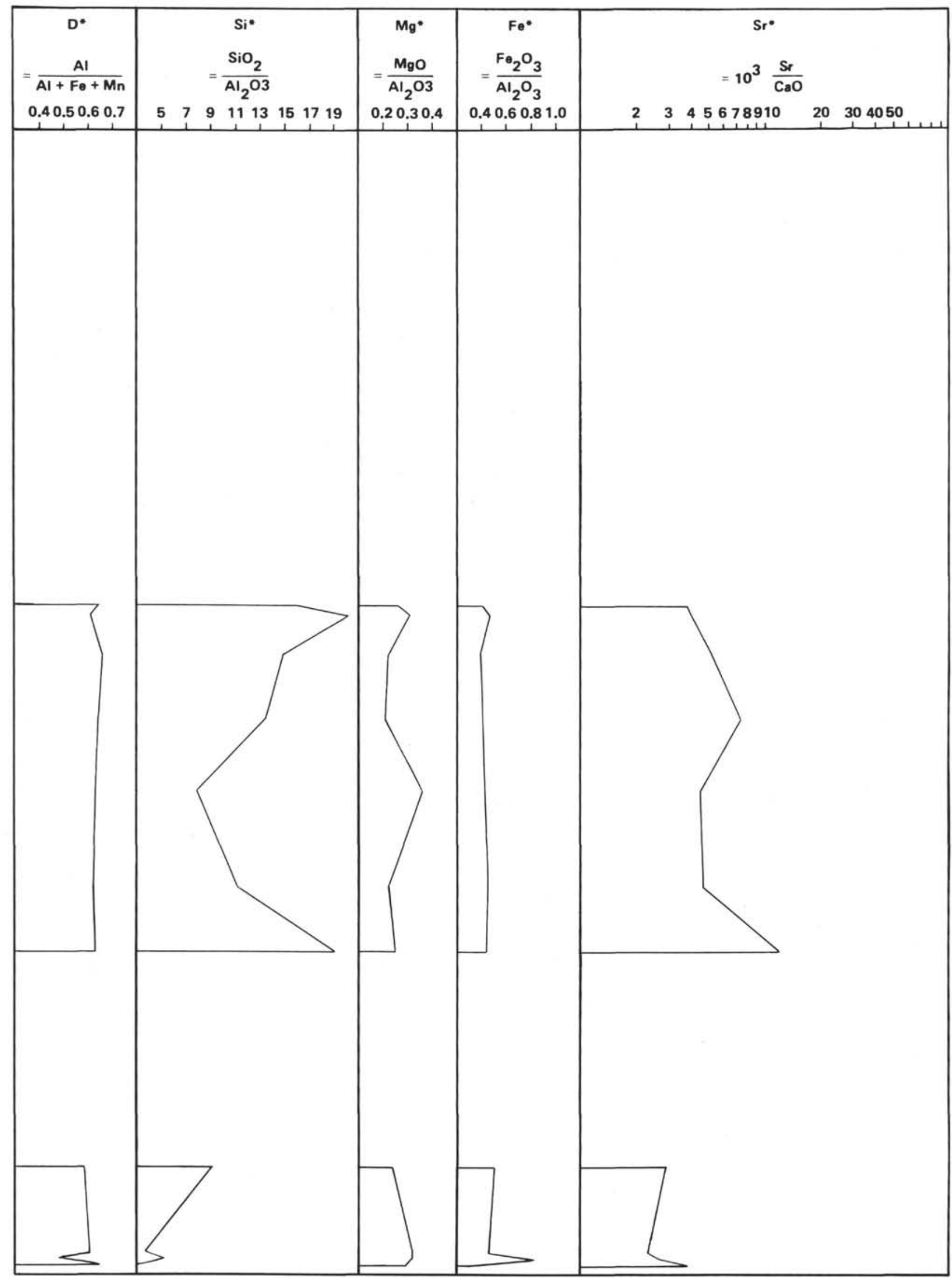

Figure 6. (Continued). 


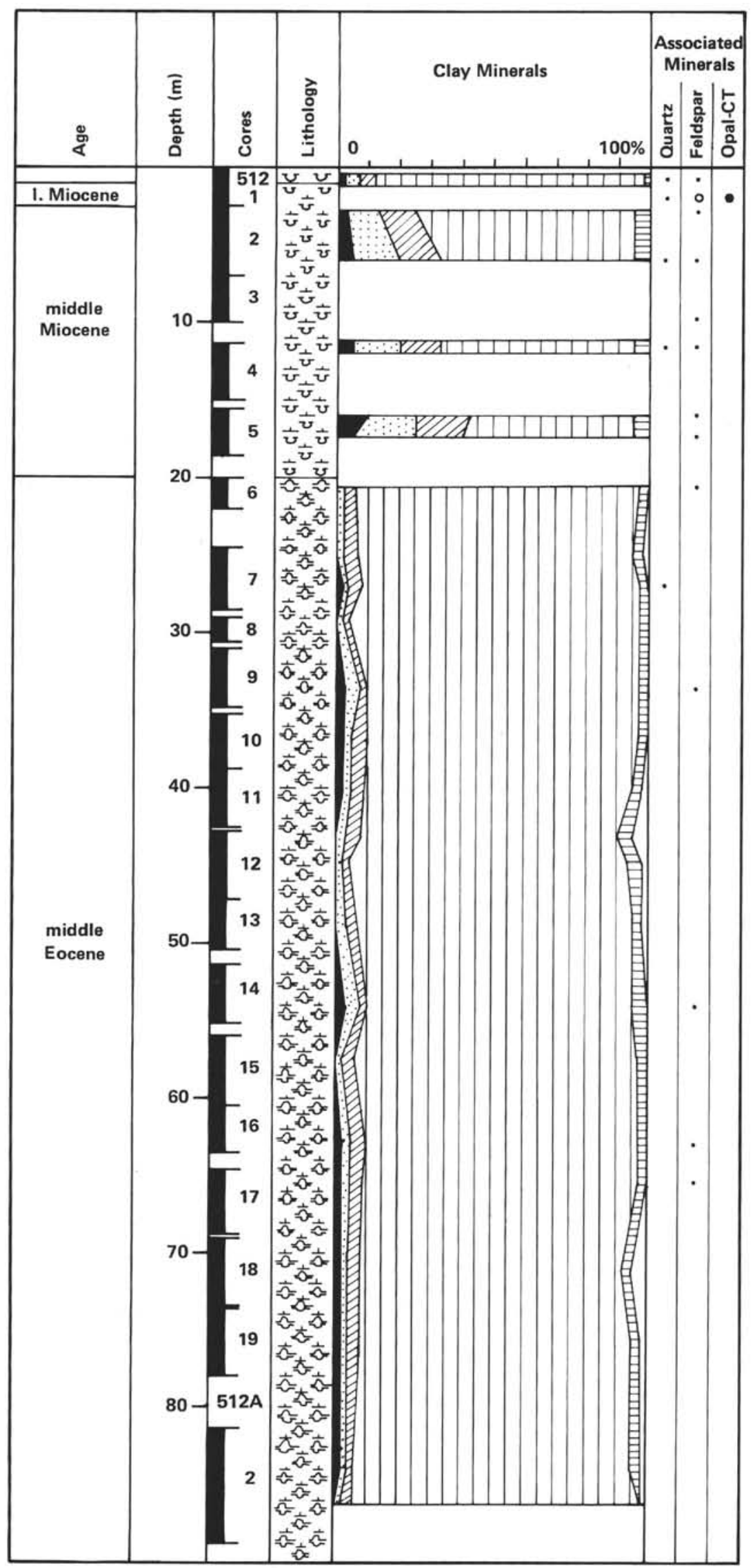

Figure 7. Holes 512 and 512A results. 


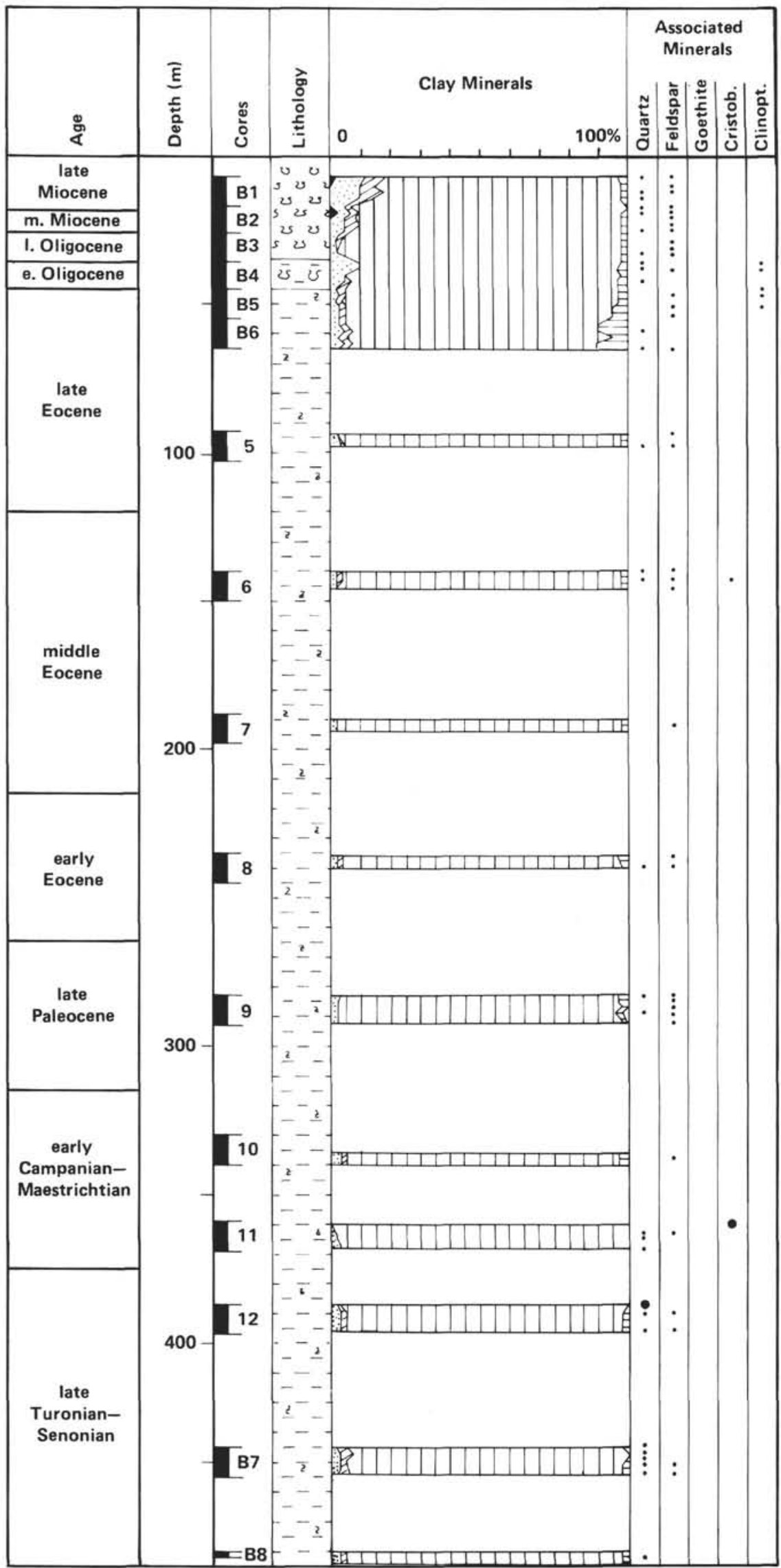

Figure 8. Holes 328 and 328B results. Cores B1-B8 are from Hole 328B. 


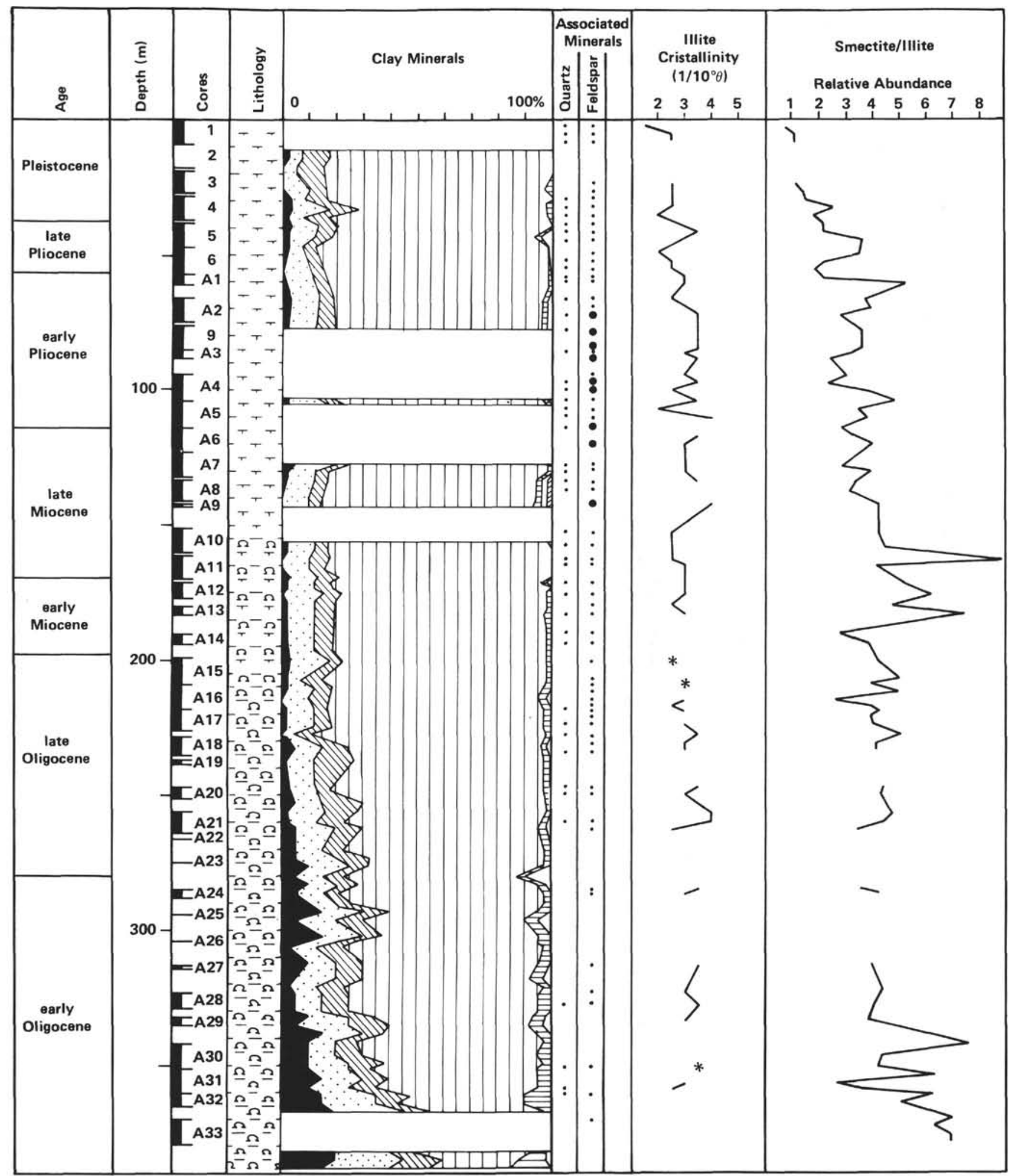

Figure 9. Holes 513 and 513A results. Cores A1-A33 are from Hole 513A.

At the end of the Jurassic, smectite abundance increased markedly. This mineral is principally derived from deep soils in downstream, flat, continental lowlands (Paquet, 1969). Its increase suggests the end or an important diminution of the earlier tectonic activity, with peneplanation of continental areas and develop- ment of smectite in the newly formed coastal plains. As in the South Atlantic basins, the greater importance of smectite corresponds to a period of stabilization of the continental margins, following the first stages of ocean basin opening. The influence of the continental environment decreases and that of shallow-water environments 


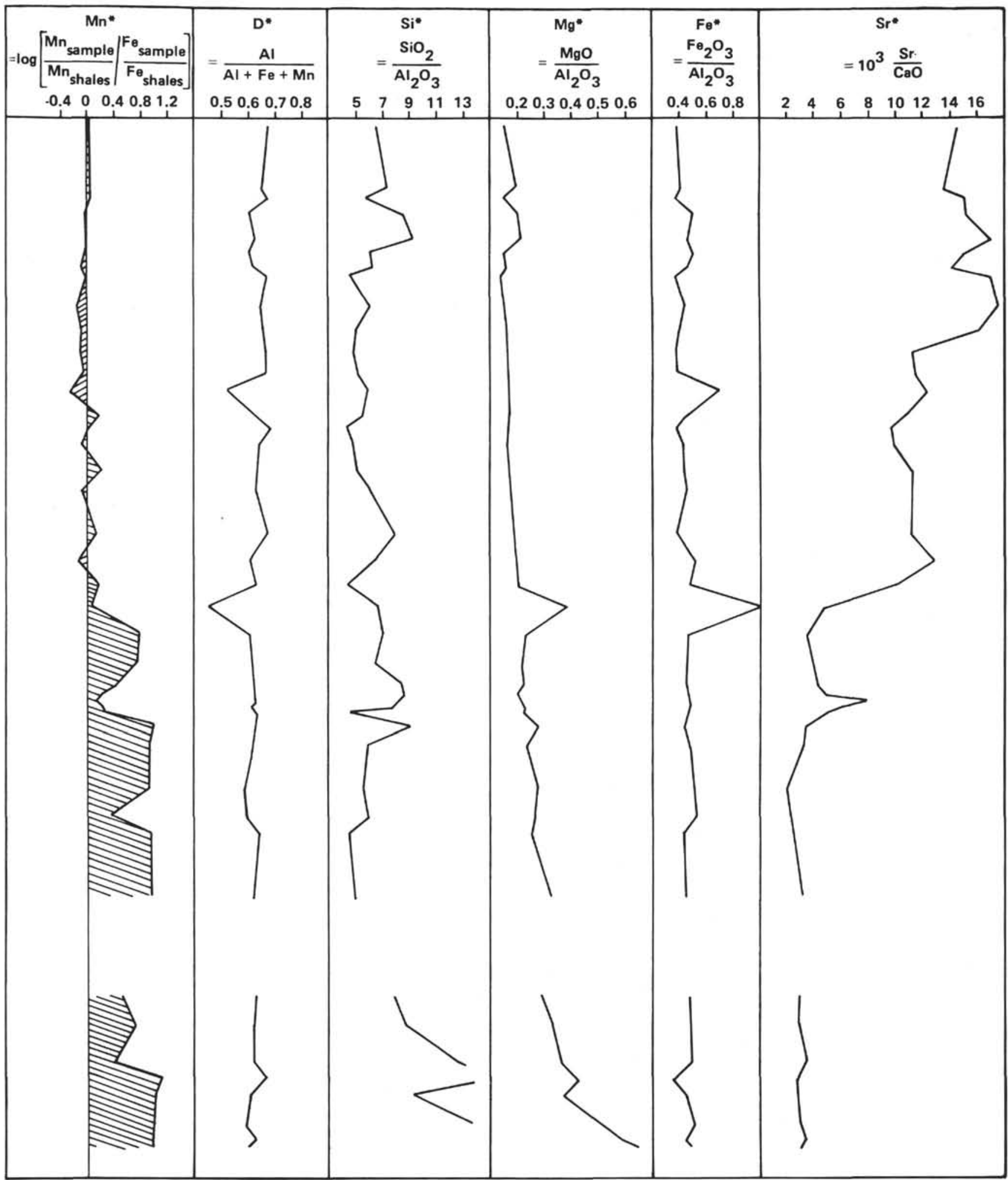

Figure 9. (Continued).

increases; reducing conditions slightly decrease. The overall importance of the detrital components remains constant. By this epoch, the separation of Africa and Antarctica was probably complete, or nearly so. Thus, tectonic quiescence appears to be characteristic of the
Falkland Plateau and Georgia Basin areas during the Late Jurassic.

From the end of the Jurassic to the Aptian/Albian boundary, chlorite, illite, and mixed-layer clays remain relatively abundant, perhaps in response to local in- 


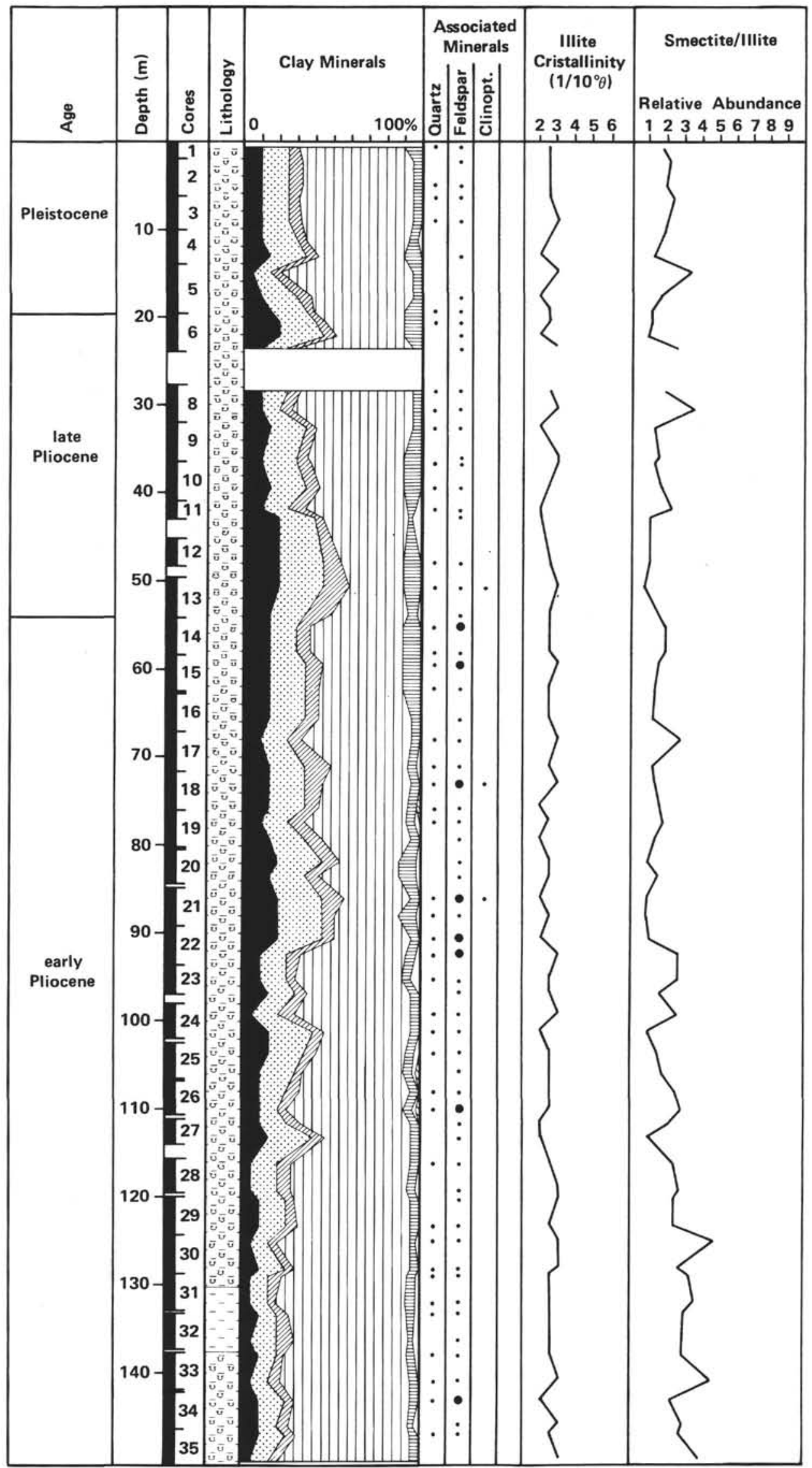

Figure 10 . Hole 514 results. 
Table 5. Site 513 geochemical results.

\begin{tabular}{|c|c|c|c|c|c|c|c|c|c|c|c|c|c|c|c|c|c|c|c|c|}
\hline $\begin{array}{c}\text { Sample } \\
\text { (level in cm) }\end{array}$ & $\begin{array}{l}\mathrm{SiO}_{2} \\
(\%)\end{array}$ & $\underset{(\%)}{\mathrm{Al}_{2} \mathrm{O}_{3}}$ & $\begin{array}{l}\mathrm{CaO} \\
(\%)\end{array}$ & $\begin{array}{l}\mathrm{MgO} \\
(\%)\end{array}$ & $\begin{array}{c}\mathrm{Na}_{2} \mathrm{O} \\
(\%)\end{array}$ & $\begin{array}{l}\mathrm{K}_{2} \mathrm{O} \\
(\%)\end{array}$ & $\begin{array}{l}\mathrm{TiO}_{2} \\
(\%)\end{array}$ & $\begin{array}{c}\mathrm{P}_{2} \mathrm{O}_{5} \\
(\%)\end{array}$ & $\begin{array}{c}\mathrm{Fe}_{2} \mathrm{O}_{3} \\
(\%)\end{array}$ & $\begin{array}{c}\text { Mn } \\
\text { (ppm) }\end{array}$ & $\underset{(\mathrm{ppm})}{\mathrm{Zn}}$ & $\begin{array}{c}\mathrm{Li} \\
(\mathrm{ppm})\end{array}$ & $\begin{array}{c}\mathrm{Ni} \\
(\mathrm{ppm})\end{array}$ & $\underset{(\mathrm{ppm})}{\mathrm{Cr}}$ & $\underset{(\mathrm{ppm})}{\mathrm{Sr}}$ & $\begin{array}{c}\text { Co } \\
\text { (ppm) }\end{array}$ & $\underset{(\mathrm{ppm})}{\mathrm{Cu}}$ & $\begin{array}{c}\mathrm{Pb} \\
(\mathrm{ppm})\end{array}$ & $\underset{(\mathrm{ppm})}{\mathrm{V}}$ & $\underset{(\mathrm{ppm})}{\mathrm{Cd}}$ \\
\hline $513-1-4,110$ & 66.20 & 10.08 & 0.94 & 1.54 & 3.75 & 1.96 & 0.44 & n.d. & 3.68 & 347 & 105 & 34 & 9 & 29 & 137 & 3 & 49 & 25 & 95 & 1 \\
\hline $3-3,80$ & 63.45 & 8.80 & 0.91 & 1.68 & 5.09 & 2.02 & 0.46 & n.d. & 3.54 & 326 & 116 & 33 & 12 & 29 & 126 & 2 & 53 & 19 & 103 & 1 \\
\hline $4-1,125$ & 62.55 & 10.98 & 1.08 & 1.67 & 4.45 & 2.32 & 0.61 & n.d. & 4.04 & 384 & 121 & 34 & 4 & 33 & 163 & 2 & 41 & 26 & 97 & 2 \\
\hline $4-5,125$ & 66.30 & 7.79 & 0.87 & 1.52 & 4.85 & 1.78 & 0.41 & n.d. & 3.86 & 316 & 89 & 27 & 1 & 23 & 132 & 0 & 40 & 26 & 84 & 1 \\
\hline $5-3,14$ & 66.10 & 7.26 & 0.77 & 1.49 & 4.87 & 1.78 & 0.40 & n.d. & 3.32 & 268 & 95 & 26 & 1 & 19 & 132 & 0 & 27 & 22 & 68 & 1 \\
\hline $6-2,14$ & 63.65 & 10.63 & 0.98 & 1.63 & 3.71 & 2.17 & 0.57 & n.d. & 5.29 & 432 & 95 & 33 & 1 & 28 & 147 & 4 & 43 & 33 & 87 & 1 \\
\hline $6-6,14$ & 64.95 & 10.21 & 1.12 & 1.63 & 4.04 & 2.12 & 0.55 & n.d. & 4.68 & 347 & 105 & 30 & 1 & 26 & 158 & 3 & 29 & 26 & 84 & 1 \\
\hline $9-2,110$ & 60.10 & 12.16 & 1.08 & 1.91 & 4.45 & 2.44 & 0.63 & n.d. & 4.90 & 353 & 111 & 37 & 5 & 32 & 174 & 6 & 36 & 33 & 113 & 2 \\
\hline $513 \mathrm{~A}-1-1,130$ & 60.60 & 13.46 & 1.15 & 1.88 & 3.98 & 2.59 & 0.70 & n.d. & 5.00 & 437 & 132 & 40 & 24 & 31 & 195 & 18 & 78 & 57 & 134 & 1 \\
\hline $2-3,34$ & 63.20 & 10.63 & 0.98 & 1.58 & 4.72 & 2.24 & 0.48 & n.d. & 4.54 & 289 & 121 & 31 & 1 & 28 & 174 & 3 & 107 & 33 & 92 & 1 \\
\hline $3-1,74$ & 59.95 & 12.40 & 1.61 & 1.98 & 4.21 & 2.41 & 0.60 & n.d. & 4.72 & 342 & 132 & 37 & 0 & 35 & 179 & 5 & 38 & 20 & 111 & 1 \\
\hline $4-1,24$ & 60.05 & 11.93 & 1.57 & 2.00 & 4.31 & 2.41 & 0.58 & n.d. & 4.68 & 400 & 89 & 38 & 0 & 35 & 179 & 7 & 51 & 29 & 100 & 1 \\
\hline $4-5,24$ & 60.30 & 10.27 & 1.22 & 1.78 & 3.08 & 2.26 & 0.54 & n.d. & 7.15 & 358 & 121 & 34 & 46 & 31 & 147 & 20 & 57 & 14 & 103 & 1 \\
\hline $5-3,25$ & 60.90 & 11.45 & 1.47 & 1.93 & 3.71 & 2.42 & 0.58 & n.d. & 5.00 & 637 & 124 & 38 & 0 & 36 & 158 & 10 & 48 & 14 & 92 & 1 \\
\hline $6-1,24$ & 58.80 & 13.34 & 1.85 & 2.11 & 3.94 & 2.56 & 0.68 & n.d. & 4.75 & 432 & 175 & 39 & 0 & 36 & 179 & 6 & 57 & 14 & 113 & 1 \\
\hline $6-5,24$ & 58.55 & 12.04 & 1.75 & 1.92 & 4.48 & 2.42 & 0.63 & n.d. & 5.00 & 384 & 126 & 36 & 0 & 37 & 174 & 13 & 67 & 15 & 105 & 1 \\
\hline $7-6,24$ & 59.95 & 12.04 & 1.61 & 2.02 & 3.81 & 2.43 & 0.58 & n.d. & 5.18 & 732 & 136 & 35 & 9 & 34 & 179 & 8 & 24 & 14 & 79 & 1 \\
\hline $8-3,90$ & 62.85 & 10.47 & 1.33 & 1.82 & 3.71 & 2.29 & 0.51 & n.d. & 4.65 & 358 & 150 & 36 & 0 & 34 & 147 & 4 & 53 & 13 & 89 & 1 \\
\hline $10-2,22$ & 66.05 & 8.50 & 1.15 & 1.51 & 4.08 & 2.20 & 0.43 & n.d. & 3.86 & 379 & 82 & 24 & 0 & 25 & 126 & 0 & 15 & 12 & 58 & 0 \\
\hline $11-2,20$ & 63.55 & 10.04 & 1.12 & 1.87 & 2.97 & 2.29 & 0.50 & n.d. & 5.00 & 332 & 103 & 38 & 7 & 33 & 142 & 3 & 82 & 15 & 79 & 1 \\
\hline $12-2,4$ & 54.65 & 13.11 & 1.85 & 2.61 & 4.15 & 2.62 & 0.60 & n.d. & 6.08 & 784 & 132 & 80 & 28 & 33 & 184 & 9 & 120 & 31 & 92 & 1 \\
\hline $13-1,8$ & 45.40 & 6.93 & 13.74 & 2.71 & 3.10 & 1.54 & 0.32 & n.d. & 6.43 & 647 & 79 & 34 & 19 & 14 & 611 & 3 & 132 & 9 & 45 & 0 \\
\hline $14-1,50$ & 29.60 & 4.27 & 28.68 & 0.98 & 2.53 & 1.04 & 0.20 & n.d. & 1.97 & 1089 & 54 & 19 & 14 & 10 & 984 & 5 & 30 & 15 & 21 & 1 \\
\hline $15-2,20$ & 30.80 & 4.85 & 26.76 & 1.04 & 2.78 & 1.20 & 0.23 & n.d. & 2.18 & 1116 & 82 & 20 & 9 & 11 & 1011 & 5 & 13 & 17 & 24 & 1 \\
\hline $16-1,21$ & 48.55 & 5.79 & 15.67 & 1.26 & 3.40 & 1.48 & 0.25 & n.d. & 2.57 & 600 & 83 & 23 & 17 & 14 & 632 & 7 & 11 & 13 & 66 & 1 \\
\hline $16-3,21$ & 61.00 & 7.14 & 7.63 & 1.40 & 2.19 & 1.70 & 0.31 & n.d. & 3.29 & 458 & 121 & 32 & 12 & 20 & 358 & 5 & 14 & 13 & 68 & 3 \\
\hline $16-5,21$ & 63.40 & 7.97 & 2.55 & 1.71 & 3.88 & 2.00 & 0.40 & n.d. & 3.68 & 400 & 113 & 35 & 12 & 25 & 200 & 4 & 14 & 12 & 84 & 1 \\
\hline $16-7,26$ & 58.35 & 7.72 & 6.54 & 1.70 & 3.27 & 1.90 & 0.35 & n.d. & 3.72 & 526 & 141 & 34 & 15 & 19 & 368 & 6 & 14 & 16 & 58 & 1 \\
\hline $17-1,47$ & 35.10 & 7.86 & 10.28 & 1.74 & 3.44 & 2.02 & 0.40 & n.d. & 3.57 & 568 & 92 & 36 & 25 & 21 & 484 & 8 & 24 & 11 & 74 & 0 \\
\hline $17-5,47$ & 26.75 & 2.95 & 31.03 & 0.80 & 2.09 & 0.78 & 0.14 & n.d. & 1.25 & 868 & 79 & 14 & 6 & 9 & 1000 & 4 & 21 & 21 & 16 & 2 \\
\hline $18-3,19$ & 16.95 & 2.95 & 38.13 & 0.69 & 1.55 & 0.69 & 0.12 & n.d. & 1.32 & 874 & 71 & 12 & 10 & 11 & 1142 & 7 & 4 & 22 & 24 & 2 \\
\hline $20-1,23$ & 16.60 & 3.14 & 37.95 & 0.85 & 1.60 & 0.78 & 0.15 & n.d. & 1.54 & 1100 & 63 & 16 & 16 & 11 & 1111 & 9 & 3 & 22 & 29 & 2 \\
\hline $21-1,143$ & 36.75 & 6.49 & 20.92 & 1.66 & 2.29 & 1.58 & 0.28 & n.d. & 3.32 & 653 & 113 & 35 & 31 & 16 & 637 & 8 & 15 & 17 & 50 & 0 \\
\hline $21-5,143$ & 11.70 & 2.69 & 41.63 & 0.68 & 1.11 & 0.64 & 0.11 & n.d. & 1.11 & 774 & 47 & 12 & 11 & 11 & 1000 & 9 & 0 & 23 & 47 & 2 \\
\hline $24-2,80$ & 8.85 & 1.90 & 45.12 & 0.60 & 1.25 & 0.51 & 0.10 & n.d. & 0.82 & 563 & 54 & 10 & 8 & 9 & 1326 & 8 & 27 & 26 & 58 & 3 \\
\hline $28-1,40$ & 26.20 & 3.45 & 31.80 & 0.95 & 1.58 & 0.90 & 0.11 & n.d. & 1.54 & 453 & 70 & 20 & 18 & 11 & 916 & 8 & 24 & 21 & 18 & 2 \\
\hline $29-2,90$ & 23.20 & 2.72 & 36.73 & 0.87 & 1.28 & 0.72 & 0.13 & n.d. & 1.25 & 542 & 59 & 20 & 6 & 12 & 989 & 4 & 3 & 23 & 18 & 2 \\
\hline $30-4,20$ & 41.80 & 3.37 & 21.69 & 1.18 & 2.48 & 0.92 & 0.11 & n.d. & 1.57 & 347 & 81 & 39 & 20 & 9 & 695 & 4 & 8 & 19 & 39 & 0 \\
\hline $31-3,15$ & 31.95 & 1.42 & 31.13 & 0.60 & 1.60 & 0.40 & 0.07 & n.d. & 0.50 & 547 & 39 & 20 & 5 & 6 & 784 & 2 & 9 & 18 & 24 & 1 \\
\hline $31-7,15$ & 18.25 & 2.02 & 39.53 & 0.75 & 1.06 & 0.55 & 0.10 & n.d. & 0.89 & 816 & 43 & 22 & 8 & 9 & 1032 & 5 & 0 & 13 & 29 & 2 \\
\hline $33-1,5$ & 21.10 & 1.56 & 36.38 & 0.78 & 1.52 & 0.45 & 0.08 & n.d. & 0.75 & 658 & 106 & 29 & 34 & 7 & 1037 & 10 & 25 & 14 & 34 & 2 \\
\hline $33-5,5$ & 29.85 & 1.54 & 32.01 & 0.90 & 1.21 & 0.45 & 0.08 & n.d. & 0.64 & 484 & 58 & 48 & 17 & 7 & 1000 & 7 & 3 & 9 & 63 & 1 \\
\hline $33-7,5$ & 30.25 & 1.35 & 31.83 & 0.86 & 1.45 & 0.42 & 0.07 & n.d. & 0.64 & 500 & 42 & 38 & 2 & 12 & 916 & 2 & 0 & 7 & 50 & 1 \\
\hline
\end{tabular}

Note: n.d. $=$ no data.

stability caused by the progressive sliding of the Falkland Plateau along the South African margin and by the distant tectonic activity associated with the opening of the Cape Basin during the Early Cretaceous (Robert et al., 1979). This abundance ended near the Aptian/Albian boundary, by which time the South Atlantic basins had opened and their margins had stabilized. A diminution in the importance of the continental environment occurred at the same time, and open marine conditions began to influence the chemical composition of the sediment. Slow subsidence of the ocean basin affected the detrital index $\left(D^{*}\right)$ : from the Coniacian to the Campanian, the importance of deeper water masses became greater, and strong, oxygen-rich currents were present by early Maestrichtian time.

During the Late Cretaceous, two small increases in chlorite, illite, mixed-layer clays, and kaolinite occurred. The first was from Albian to Turonian time, the second during Coniacian-Santonian times. These mineralogical events suggest the occurrence of minor tectonic activity nearby or of major, distant, tectonic events. Uplift or emergence of the Maurice Ewing Bank are not evident from clay mineralogical and geochemical data, despite the presence of Inoceramus fragments, indicators of shallow-water environment, at Site 511. It appears that the mineralogical increases are contemporaneous with the first Andean deformational phases-a collision event between southernmost South America and the Antarctic Peninsula - and migration of a Pacific island arc toward South America (Dalziel et al., 1974; De Wit, 1977).

In the early Paleocene, a minor increase in chlorite, illite, and mixed-layer clays occurred at Site 327, suggesting a minor rejuvenation of continental relief. This event is missing at Site 511, where a hiatus occurred, and in the Georgia Basin, where only smectite is present. The mineralogical change that occurred at the Cretaceous/Tertiary boundary results mainly from a marginal tectonic rejuvenation and secondarily from increased oceanic circulation; both probably originated from a major phase of ocean basin opening (Chamley and Robert, 1979). These mineralogical changes were locally important-as they were, for instance, on the Rio Grande Rise (Robert, 1981)-but were of very minor importance in the Argentine Basin and were practically absent in the Cape Basin and on the Falkland Plateau. An orogenic episode located in the Antarctic Peninsula and Southern Andes (Termier and Termier, 1957) could explain the minor mineralogical changes at Site 327.

Paleocene and Eocene sediments are characterized by smectite, which indicates a period of tectonic quiescence on the continental land masses adjacent to the Falkland Plateau region. 


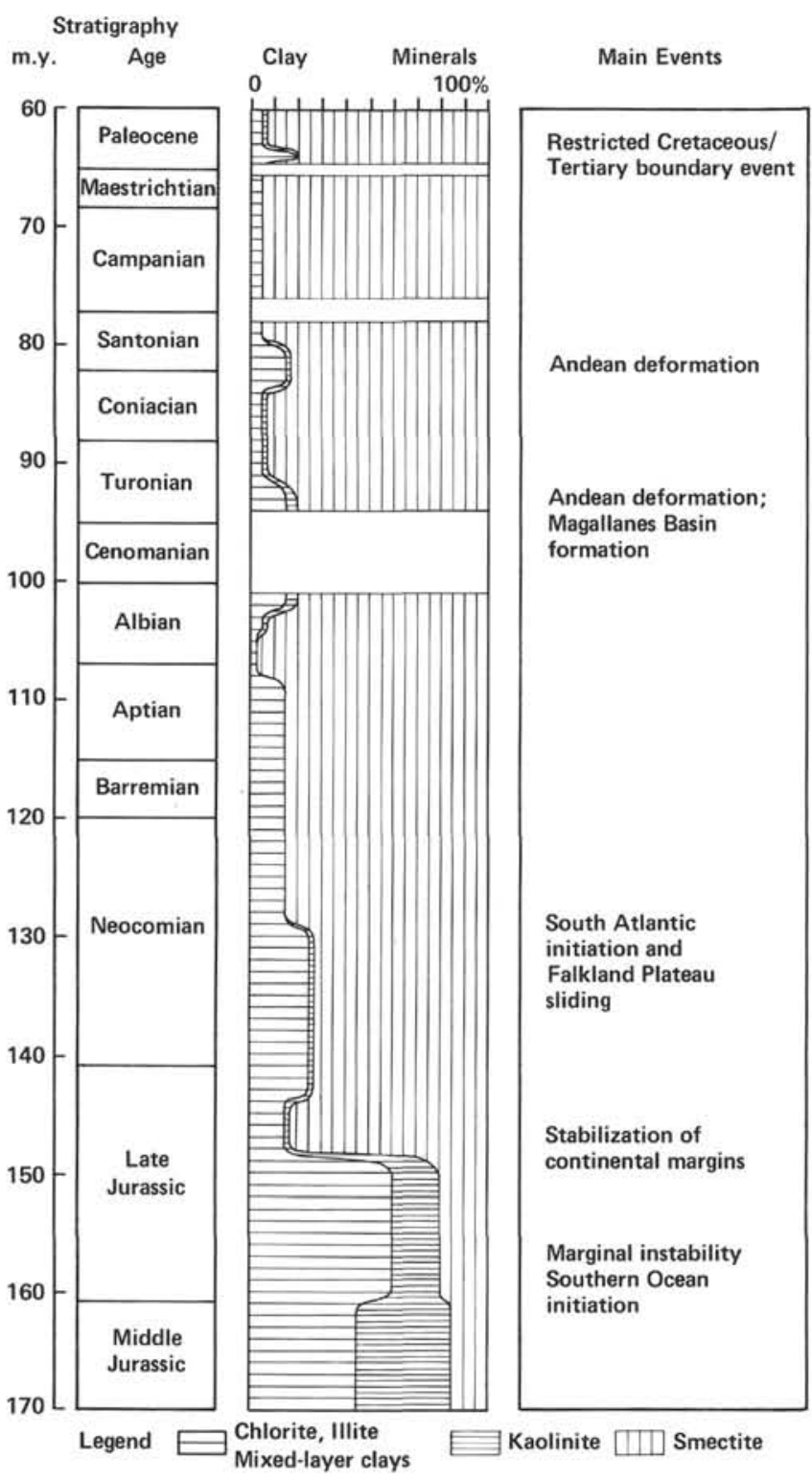

Figure 11. Evolution and significance of Mesozoic clay sediments on the Maurice Ewing Bank, Holes 327A, 330, and 511.

\section{MARINE PALEOENVIRONMENTAL AND CLIMATIC EVOLUTION DURING CENOZOIC TIME}

\section{Influence of Antarctic Bottom Water $(\mathrm{AABW})$ on the Clay Sedimentation}

In the South Atlantic realm from the late Eocene cooling onward, the influences of climate and currents on the clay mineral distribution became very important (Robert, 1980) and progressively masked the tectonic and morphologic influences. Continuously less intense continental weathering resulted in a minor development of soils and in an increase of chlorite, illite, and mixedlayer clays in detrital sediments (Chamley, 1979). The composition of the clay fraction became increasingly similar to that of present-day sediments.
Generally, the cooling expressed by the clay particles appears more marked toward high latitudes. In the sediments recovered during Legs 36 and 71 , smectite remains abundant compared to the Cape Basin, Walvis Ridge, or Rio Grande Rise (Robert, 1980). Moreover, chlorite, illite, and mixed-layer clays are more abundant on the Maurice Ewing Bank, an elevated feature, than in the Georgia Basin (Site 328) or on the Mid-Atlantic Ridge (Sites 513, 514), where deeper sites were drilled. These sites are located in oceanic regions subjected to AABW currents (Le Pichon et al., 1971). In the Vema Channel, the abundance of smectite in the sediments underlying AABW has been demonstrated (Chamley, 1975; Melguen et al., 1978a).

The mineralogical studies carried out on marine sediments in deglaciated Antarctic coastal areas show principally the presence of chlorite and illite (Kagami, 1964; Michel, 1964; Chamley, 1965); at present, weathering does not permit the formation of smectite, although small amounts of the mineral were found in some sediments studied by Kagami (1964). Smectite has been found, however, in the sediments recovered during DSDP Legs 28 (Ross Sea) and 35 (Bellingshausen Sea), where it sometimes represents more than $50 \%$ of the clay fraction (Cook et al., 1975; Zemmels and Cook, 1976). The Ross Sea is an important source of the AABW which flows through the Bellingshausen Sea. Thus, it seems that the Antarctic continent is directly involved in the detrital supply of smectite to regions subjected to AABW.

Smectite is not derived from weathering on the glaciated Antarctic land mass; it may result from erosion of Mesozoic or early Cenozoic sediments outcropping on or around Antarctica. Such a mechanism has been proposed by Kemp (1972) to explain the occurrence of Mesozoic and early Cenozoic palynomorphs in bottom sediments from the continental shelf around Antarctica. Unfortunately, mineralogical studies of the Mesozoic and early Cenozoic sediments, as well as of the volcaniclastics which have been described by Cailleux (1963) and Warren (1965), have not yet been carried out. In the Northern Hemisphere, clay mineralogical studies of bottom sediments from the Arctic Ocean indicated the presence of kaolinite derived from ancient shales of Northern Alaska and Canada (Naidu et al., 1971; Darby, 1975).

In the region studied on Legs 36 and 71 , smectite is probably derived mainly from the Weddell Sea area, a major source of AABW for the Atlantic Ocean (Gordon, 1971; Ledibetter and Ciesielski, 1982). AABW is produced beneath an extensive ice-shelf formed by the coalescence of glaciers that drain and erode a large area of Antarctica. During Plio-Pleistocene time, clay particles may also have originated in the Weddell Basin, where erosion by AABW has removed all sediment younger than early Pliocene, in many areas exposing older sediments (Ledbetter and Ciesielski, 1982).

Another possibility for the origin of detrital smectite is the Falkland Plateau itself, where Mesozoic and Cenozoic sediments crop out. Intense erosion started during the Miocene with the initiation of the Antarctic 
Circumpolar Current (ACC). Differences in the smectite abundance between elevated and deeper sites became significant near the Eocene/Oligocene boundary, when a pre-AABW probably developed (Ciesielski et al., 1982). On the Maurice Ewing Bank, Plio-Pleistocene sediment shows an increase in the abundance of smectite at a time when the ACC, which had previously truncated the sedimentary sequence on the Bank, diminished in intensity, permitting another brief period of sedimentary deposition (Ciesielski and Wise, 1977). Thus, beginning with the late Miocene erosion, the Falkland Plateau may have supplied sediments rich in smectite for distant redeposition, as shown by Ciesielski et al. (1982.)

Volcanic Influence on the Rift, Carbonate Dissolution, and the Opening of the Drake Passage

In the Oligocene sediments at Site 513, above the basaltic basement, a strong increase in the concentration of transitional elements occurs (Table 5). These elements remain abundant until the early Miocene, when their amounts decrease. By the same time, however, a strong increase in the sedimentation rate masks the diminution in the real abundance of the transitional elements that occurred when the site edged away from the rift area. A similar effect has been shown at Site 19 on the MidAtlantic Ridge (Maillot and Robert, 1980).

From lower Oligocene to lower upper Oligocene sediments a low $\mathrm{Sr}^{*}$, derived from marine carbonates is typical; it suggests that Site 513 was located above the paleolysocline. At the top of the upper Oligocene and in the lower Miocene, a small increase in $\mathrm{Sr}^{*}$ is probably related to a slight dissolution of carbonates; by this time, the site was probably situated below the lysocline and above the CCD. From the top of the lower Miocene upward, $\mathrm{Sr}^{*}$ strongly increases, in response to the passage of Site 513 through the CCD.

During early Miocene time, a break occurs in the geochemical indexes (Fig. 9). Below this break, as during Oligocene time, the sediments are characterized by both detrital and volcanogenic influences. Above the break, several changes occur in the sedimentation. In addition to the increase in $\mathrm{Sr}^{*}$ related to the deepening of Site 513 , a strong decrease of $\mathrm{Mn}^{*}$ and a slight increase of $\mathrm{D}^{*}$ correspond to a dilution of the volcanic influences of the rift by terrigenous elements. These geochemical events occur contemporaneously with the opening of the Drake Passage (Barker and Burrell, 1977), which is not clearly recognizable in the clay mineralogy. However, fluctuations appear in the S/I of Site 513 at this time, and persist until the beginning of the late Miocene. These variations could result from changes in current velocity, since the opening of the Drake Passage was marked by intense scouring and removal of sediments, as evidenced on the Maurice Ewing Bank (Ciesielski and Wise, 1977). In Core 11 of Hole 513A, the reworking of Eocene sediments revealed by a study of silicoflagellates is also recognizable by a sharp increase of the $\mathrm{S} / \mathrm{I}$ index. At Site 329, a large decrease in the S/I index occurred from the Oligocene to the early middle Miocene, contemporaneously with the opening of Drake Passage
(Fig. 6); the significance of this break is not evident at the present time.

\section{Influence of Climate on the Clay Sedimentation}

The first influence of Cenozoic climatic cooling on the clay minerals was recorded during the late Eocene at Site 511, consistent with the data of Kennett (1977) and Mercer (1978). The general evolution of the Cenozoic glaciation is well expressed by the $\mathrm{S} / \mathrm{I}$ index, shown in Figures 6, 9, and 10 for Sites 329, 513, and 514 only.

In the lower Oligocene, there is a progressive decrease in the $\mathrm{S} / \mathrm{I}$ index upwards from the base of the sedimentary section. By the early Oligocene, the deterioration of climate had resulted in the development of modern deep-water masses and a more intense oceanic circulation (van Andel et al., 1977; Moore et al., 1978).

During the late Miocene, a decrease occurred in the $\mathrm{S} / \mathrm{I}$ index, corresponding to the time during which the Antarctic ice-sheet developed (Shackleton and Kennett, 1975). This decrease is present at both Sites 329 and 513. Another S/I ratio change occurred during the Pliocene, at the beginning of the Gauss Magnetic Epoch, at a time when a second strong cooling induced an increase in the Antarctic ice-sheet (Watkins and Kennett, 1971; Mercer, 1978) and initiated extensive glaciation over Patagonia (Mercer, 1976).

Near the Pliocene/Pleistocene boundary, a major decrease in the S/I occurred at Site 513 and a slight increase at Site 514. During Pliocene times, the abundance of smectite in the clay fraction demonstrates that both sites were under the influence of AABW or circumpolar deep water; this influence was probably felt more strongly at Site 513. At the end of the Pliocene, smectite abundance decreased at Site 513, and the mineralogical composition of the clay fraction became more similar to that of Recent, high-latitude, detrital sediments (Biscaye, 1965; Griffin et al., 1968; Froget, in press). At Site 514, the increase in the abundance of smectite probably expresses an increased AABW flow favoring the transport and redeposition of smectite particles. These data suggest a change in AABW circulation at a time when a decrease in AABW production has been noted by Ledbetter and Ciesielski (1982).

Some paleoclimatic features, such as the early-middle Miocene warming reported by Margolis et al. (1977), are not recorded by the clay minerals, probably because currents exercised an important influence in this region.

\section{CONCLUSIONS}

In the South Atlantic, clay mineralogical data are chiefly influenced by climate. During Mesozoic times, the relative persistence of a globally warm climate, with contrasts in humidity, permits an interpretation of the morphologic and tectonic evolution of the continental land masses: the initiation of the ocean basins, stabilization of the continental margins, major phases of ocean widening, and inferred tectonic events. During Cenozoic time, the importance of these influences decreased as the climate began to cool. By this time, clay mineralogical data express different phases in the degradation of climate; from them we can also infer variations in 
currents the initiation of the bottom-water masses and changes in their circulation, and the development of Antarctic and Patagonian glaciations. Geochemical data express principally the modification of the oceanic environment: the evolution from a continental to a reduced marine and then to an open marine environment, the influences of marine currents, volcanogenic influences of the rift, carbonate dissolution, and the deepening of the ocean basins. Correlated mineralogical and geochemical studies can thus help to reconstruct paleoenvironments.

\section{ACKNOWLEDGMENTS}

Thanks are due to the U.S. National Science Foundation for samples from Legs 36 and 71 . Financial support was provided by CNEXO (France) for geochemical studies. Technical assistance was provided by $\mathrm{M}$. Acquaviva and C. H. Froget for clay mineral analysis and illustrations, C. Maillot for geochemical analysis; F. Dujardin typed the manuscript. B. Bornhold, H. Chamley, P. Debrabant, G. Jones, and S. Wise reviewed and improved the manuscript.

\section{REFERENCES}

Angino, E. E., 1964. Trace elements in Antarctic bottom sediments. U.S. Antarc. Proj. Office Bull., 5:164-166.

Barker, P. F., and Burrell, J., 1977. The opening of Drake Passage. Mar. Geol., 25:15-34.

Barker, P. F., Dalziel, I. W. D., et al., 1977. Init. Repts. DSDP, 36: Washington (U.S. Govt. Printing Office).

Bausch, W. M., 1968. Outlines of distribution of strontium in marine limestones. In Müller, G., and Friedman, G. M. (Eds.), Recent Developments in Carbonate Sedimentology in Central Europe: Berlin (Springer Verlag), pp. 106-115.

Biscaye, P. E., 1965. Mineralogy and sedimentation of recent deepsea clay in the Atlantic Ocean and adjacent seas and oceans. Geol. Soc. Am. Bull., 76:803-832.

Boström, K., 1970. Submarine volcanism as a source of iron. Earth Planet. Sci. Lett., 9:348-384.

Bostrom, K., Joensuu, O., Valdes, S., Charm, W., and Glaccum, R., 1976. Geochemistry and origin of East Pacific sediments samples during DSDP leg 34. In Yeats R. S., Hart S. R., et al., Init. Repts. $D S D P, 34$ : Washington (U.S. Govt. Printing Office), 559-574.

Boström, K., Joensuu, O., Valdes, S., and Riera, M., 1972. Geochemical history of South Atlantic ocean sediments since late Cretaceous. Mar. Geol., 12:85-121.

Boström, K., and Peterson, M. N., 1969. The origin of the aluminium poor ferromanganoan sediments in areas of high heat flow in the east Pacific rise. Mar. Geol., 7:427-447.

Cailleux, A., 1963. Géologie de l'Antarctique: Paris (Sedes).

Chamley, H., 1965. Observations sur quelques sédiments marins préléves près des côtes de Terre Adélie (Antarctique), Rec. Trav. Station Marine d'Endoume, 36:215-228.

1971. Recherches sur la Sédimentation Argileuse en Méditerranée. 'Sci. Geol. Mem., 35.

1975. Influence des courants profonds au large du Brésil sur la sédimentation argileuse récente. 9ème Congr. Int. Sédimentol. Nice, 8:13-21.

1979. North Atlantic clay sedimentation and paleoenvironment since the Late Jurassic. In Talwani M., Hay, W., and Ryan, W. B., F., (Eds.), Deep Drilling Results in the Atlantic Ocean: Continental Margins and Paleoenvironment: Washington, D. C. (Am. Geophys. Union), pp. 342-360.

Chamley, H., and Robert, C., 1979. Late Cretaceous to early Paleocene environmental evolution expressed by the Atlantic clay sedimentation. In Christense, W. K., and Birkelund, T. (Eds.), Cretaceous-Tertiary Boundary Events Symposium (Vol. 2): Copenhagen, Proceedings, pp. 71-77.

, in press. Paleoenvironmental significance of clay deposits in Atlantic black-shales. Cretaceous Res.

Ciesielski, P. F., Ledbetter, M. T., and Ellwood, B. B., 1982. The sedimentary and paleoceanographic record of Southern Ocean rises, Part I. The Maurice Ewing Bank. Mar. Geol., 46:1-51.

Ciesielski, P. F., and Wise, S. W., 1977. Geologic history of the Maurice Ewing Bank of the Falkland Plateau (S.W. Atlantic sector of the Southern Ocean) based on piston and drill cores. Mar. Geol., 25:175-207.

Cook, H. E., Zemmels, I., and Matti, J. C., 1975. X-ray mineralogy data, Austral-Antarctic region, Leg 28, Deep Sea Drilling Project. In Hayes, D. E., Frakes, L. A., et al., Init. Repts. DSDP, 28: Washington (U.S. Govt. Printing Office), 999-1008.

Craddock, C., 1970. Antarctic geology and Gondwanaland. Antarct. J., 5:53-57.

Dalziel, I. W. D., De Wit, M. J., and Palmer, K. F., 1974. Fossil marginal basin in the southern Andes. Nature, 250:291-294.

Darby, D. A., 1975. Kaolinite and other clay minerals in Arctic Ocean sediments. J. Sediment. Petrol., 45:272-279.

De Wit, M. J., 1977. The evolution of the Scotia arc as a key tothe reconstruction of southwestern Gondwanaland. Tectonophysics, 37: 53-81.

Dunoyer de Segonzac, G., 1969. Les Minéraux Argileux dans la Diagenèse. Passage au métamorphisme. Sci. Geol. Mem. 29.

Elderfield, H., 1977. The form of manganese and iron in marine sediments. In Glasby G. P. (Ed.), Marine Manganese Deposits: Amsterdam (Elsevier), pp. 269-290.

Frakes, L. A., 1979. Climates Throughout Geologic Time: Amsterdam (Elsevier).

Froget, C., in press. La sédimentation argileuse depuis l'Eocène sur le plateau Vøring et à son voisinage, d'après le leg 38 DSDP (mer de Norvège). Sedimentology.

Goldberg, E. D., and Griffin, J. J., 1964. Sedimentation rates and mineralogy in the South Atlantic. J. Geophys. Res., 69:4293-4309.

Gordon, A. L., 1971. Oceanography of Antarctic waters. In Reid, T. L. (Ed.), Antarctic Oceanology, Pt. 1. Amer. Geophys. Union, Antarct. Res. Ser., 15:169-203.

Griffin, J. J., Windom, H., and Goldberg, E. D., 1968. The distribution of clay minerals in the world ocean. Deep Sea Res., 15: 433-460.

Herbin, J. P., and Deroo, G., 1979. Etude sédimentologique de la matière organique dans les argiles noires crétacées de l'Atlantique Sud. Doc. Lab. Geol. Fac. Sci. Lyon, 75:71-87.

Kagami, H., 1964. A description of bottom sediments in LützoroHolmbukta. In Adie, R. J., (Ed.), Antarctic Geology: Amsterdam (North-Holland), pp. 703-709.

Kemp, E. M., 1972. Recycled palynomorphs in continental shelf sediments from Antarctica. Antarctic J., 7:5.

Kennett, J. P., 1977. Cenozoic evolution of Antarctic glaciation, the Circum-Antarctic Ocean, and their impact on global paleoceanography. J. Geophys. Res., 82:3843-3860.

Krishnaswami, S., 1976. Authigenic transition elements in Pacific pelagic clays. Geochim. Cosmochim. Acta, 40:425-434.

Ledbetter, M. T., and Ciesielski, P. F., 1982. Bottom current erosion in the South Atlantic sector of the Southern Ocean. Mar. Geol., 46́:329-341.

LePichon, X., Eittreim, S. L., and Ludwig, W. J., 1971. Sediment transport and distribution in the Argentine basin. 1. Antarctic bottom current passage through the Falkland fracture zone. In Ahrens, L. H., Press, F., Runcorn, S. K., and Urey, H. C., (Eds.), Physics and Chemistry of the Earth: Oxford (Pergamon Press), pp. 3-27.

Lisitzin, A. P., 1962. Bottom sediments of the Antarctic. In Wexler, H., Rubin, M. J., and Caskey, J. E. (Eds.), Antarctic Research. Geophys. Monograph., 7:81-88.

Maillot, H., 1979. Etude géochimique de la marge Sud-Est et Atlantique (Leg 40 DSDP). Tème Réun. Ann. Sci. Terre, Lyon (Soc. géol. France), p. 306.

1980. Géochimie des sédiments crétacés et cénozoïques de la marge atlantique Sud-Orientale (Leg 40 DSDP). Rev. Geol. Dyn. Geogr. Phys., 22:75-83.

, in press. Géochimie de la fraction inférieure à deux micromètres dans les sédiments de l'Atlantique Sud. Bull. Soc. Geol. Nord.

Maillot, H., and Robert, C., 1980. Mineralogie et géochimie des sédiments crétacés et cénozoïques de l'Océan Atlantique Sud (marge africaine, dorsale médio-atlantique). Bull. Soc. Geol. Fr., 22: 777-788.

Margolis, S. V., Kroopnick, P. M., and Goodney, D. E., 1977. Cenozoic and late Mesozoic paleoceanographic and paleoglacial history recorded in circum-Antarctic deep-sea sediments. Mar. Geol., 25: 131-148. 
Maxwell, A. E., Von Herzen, R. P., et al., 1970. Init. Repts. DSDP, 3: Washington (U.S. Govt. Printing Office).

Melguen, M., Debrabant, P., Chamley, H., Maillot, H., Hoffert, M., and Courtois, C., 1978a. Influence des courants profonds sur les faciès sédimentaires du Vema Channel (Atlantique Sud) à la fin du Cénozoïque. Bull. Soc. Geol. Fr., 20:121-136.

Melguen, M., Le Pichon, X., and Sibuet, J. C., 1978b. Paléoenvironment de l'Atlantique Sud. Bull. Soc. Geol. Fr., 20:471-489.

Mercer, J. H., 1976. Glacial history of southernmost South America. Quat. Res., 6:125-166.

1978. Glacial development and temperature trends in the Antarctic and in South America. In Van Zinderen Bakker, E. M., (Ed.), Antarctic Glacial History and World Paleoenvironments: Rotterdam (A. A. Balkema), pp. 73-93.

Michel, J. P., 1964. Contribution à l'Étude Sedimentologique de l' Antarctique: Paris (Comité National Français des Recherches Antarctiques), 5.

Millot, G., 1964. Géologie des Argiles: Paris (Masson).

Moore, T. C., van Andel, Tj. H., Sancetta, C., and Pisias, N., 1978. Cenozoic hiatuses in pelagic sediments. Micropaleontology, 24: 113-138.

Müller, G. and Friedman, G. M., 1968. Recent Developments in Carbonate Sedimentology in Central Europe: Berlin (Springer Verlag), pp. 106-115.

Naidu, A. S., Burrell, D. C., and Hood, D. W., 1971. Clay mineral composition and geologic significance of some Beaufort Sea sediments. J. Sediment. Petrol., 41:691-694.

Nekrassov, B., 1966. Chimie Minérale: Généralités et Étude des Éléments: Moscow (Editions de Moscou).

Paquet, H., 1969. Evolution géochimique des minéraux argileux dans les altérations et les sols des climats méditerranéens et tropicaux à saisons constratées. Sci. Geol. Mem., 30.

Pedro, G., 1968. Distribution des principaux types d'altération chimique à la surface du globe. Présentation d'une esquisse géographique. Rev. Geogr. Phys. Geol. Dyn., 10:457-470.

Robert, C., 1980. Climats et courants cénozoïques dans l'Atlantique Sud d'après l'étude des minéraux argileux (Legs 3, 39 et 40 DSDP). Oceanol. Acta, 3:369-376.
1981. Santonian to Eocene paleogeographic evolution of the Rio Grande Rise (South Atlantic) deduced from clay mineralogical data (DSDP Legs 3 and 39). Paleogeogr. Paleoclimatol. Paleoecol., 33:311-325.

Robert, C., Herbin, J. P., Deroo, G., Giroud d'Argoud, G., and Chamley, H., 1979. L'Atlantique Sud au Crétacé d'après l'étude des minéraux argileux et de la matière organique (Legs 39 et 40 DSDP). Oceanol. Acta, 2:209-218.

Shackleton, N., and Kennett, J. P., 1975. Paleotemperature history of the Cenozoic and the initiation of Antarctic glaciation: Oxygen and carbon isotope analyses in DSDP Sites 277,279 , and 281 . In Kennett, J. P., Houtz, R. E., et al., Init. Repts. DSDP, 29: Washington (U.S. Govt. Printing Office), 743-756.

Sigl, W., Chamley, H., Fabricius, F., Giroud d'Argoud, G., and Müller, J., 1978. Sedimentology and environmental conditions of sapropels. In Hsü, K. J., Montadert, L., et al., Init. Repts. DSDP, 42, Pt. 1: Washington (U.S. Govt. Printing. Office), 445-465.

Termier, H., and Termier, G., 1957. L'évolution de la Lithosphère. 2. Orogenèse: Paris (Masson).

Thompson, R. W., 1977. Mesozoic sedimentation on the eastern Falkland Plateau. In Barker, P. F., Dalziel, I. W. D., et al., Init. Repts. DSDP, 36: Washington (U.S. Govt. Printing Office), 877-892.

Turekian, K. K., 1965. Some aspects of the geochemistry of marine sediments. In Riley, J. P., and Skirrow, G., (Eds.), Chemical Oceanography: London (Academic Press), 2:81-126.

van Andel, Tj. H., Thiede, J., Sclater, J. G., and Hay, W. W., 1977. Depositional history of the South Atlantic Ocean during the last 125 million years. J. Geol., 85:651-698.

Warren, G., 1965. Geology of Antarctica. In Hatherton, T., (Ed.), Antarctica: London (Methuen and Co.) pp. 279-320.

Watkins, N. D., and Kennett, J. P., 1972. Regional sedimentary disconformities and upper Cenozoic changes in bottom water velocities between Australia and Antarctica. Am. Geophys. Union Antarct. Res. Ser., 19:273-294.

Zemmels, I., and Cook, H. E., 1976. X-ray mineralogy data from the Southeast Pacific basin, Leg 35 Deep Sea Drilling Project. In Hollister, C. D., Craddock, C., et al., Init. Repts. DSDP, 35: Washington (U.S. Govt. Printing Office), 747-754. 